\title{
War Karl Meichelbeck ein „deutscher Mauriner“?
}

\section{Thomas Stockinger}

\section{Geschichte eines Forschungskonzepts}

Der Begriff eines „deutschen Maurinismus“ ist nicht ganz neu; die Formulierung findet sich erstmals vor gut achtzig Jahren bei Paul Muschard, der in seiner - sehr breit gefassten - Geschichte der benediktinischen Kanonistik die von ihm dargestellten „historischen Schulen“, namentlich jene von St. Emmeram zu Regensburg, St. Blasien und Melk, allgemein in den Zusammenhang eines „Maurinismus“ stellte. In Bezug auf Bernhard Pez von Melk sprach er speziell von einem „deutschen Maurinismus“, dessen „literarischer Mittelpunkt“ Pez zu seiner Zeit gewesen sei ${ }^{1}$. Die Wortschöpfung Muschards brachte freilich kein neues Konzept auf, sondern ordnete sich in eine bestehende Perspektive ein, welche der Maurinerkongregation ${ }^{2}$ eine prägende und leitende Vorbildwirkung für gelehrte, insbesondere für historiographische Bestrebungen der deutschen Benediktiner im frühen 18. Jahrhundert zuschrieb ${ }^{3}$. Die meist katholischen, teils selbst benediktini-

1 Paul Muschard, Das Kirchenrecht bei den deutschen Benediktinern und Zisterziensern des 18. Jahrhunderts. Studien und Mitteilungen zur Geschichte des Benediktiner-Ordens und seiner Zweige 47 (1929) 225-315, 477-596, hier 494 (St. Emmeram), 499f. (St. Blasien), 525 (Bernhard Pez).

2 Zur allgemeinen Geschichte der Kongregation: Yves Chaussy, Les Bénédictins de Saint-Maur (Collection des Études Augustiniennes, Série Moyen-âge et Temps modernes 23-24, 2 Bde., Paris 1989-1991).

3 Joseph BADER, Das ehemalige Kloster Sanct Blasien auf dem Schwarzwalde und seine Gelehrten-Akademie. Freiburger Diöcesan-Archiv 8 (1874) 103-253; Joseph Anton ENDRES, Korrespondenz der Mauriner mit den Emmeramern und Beziehungen der letzteren zu den wissenschaftlichen Bewegungen des 18. Jahrhunderts (Stuttgart-Wien 1899); Ignaz E. KATHREIN, Aus dem Briefverkehr deutscher Gelehrten mit den Benedictinern der Congregation von St. Maur und deren Beziehungen zu den literarischen und religiösen Bewegungen des 18. Jahrhunderts. Studien und Mittheilungen aus dem Benedictiner- und dem Cistercienser-Orden mit besonderer Berücksichtigung der Ordensgeschichte und Statistik 23 (1902) 111-126, 386403, 625-631; 24 (1903) 175-184, 446-466; Eduard Ernst KATSCHTHALER, Über Bernhard Pez und dessen Briefnachlass. Jahresbericht des $k$. k. Obergymnasiums zu 
schen Forscher, die sich hiermit beschäftigten, konnten dabei ihrerseits an die seit dem 18. Jahrhundert bestehende Kontinuität der Würdigung Jean Mabillons in seinem Wirken für die historischen Hilfswissenschaften anknüpfen und dafür auf jeder katholischen Apologetik unverdächtige Autoritäten der deutschen Historikerzunft wie Wegele ${ }^{4}$ oder Wattenbach ${ }^{5}$ verweisen. Mit ihren Forschungen wurde allerdings der Blick über die engen Grenzen der Paläographie und Diplomatik hinaus erweitert, wenn etwa Joseph Anton Endres auf die Vermittlung von positiver Theologie und kartesianischer Philosophie in den Kontakten St. Emmerams mit den Maurinern hinwies ${ }^{6}$.

Aufgegriffen und mehrfach an prominenter Stelle verwendet wurde Muschards Begriff in den 1970er Jahren von Ludwig Hammermayer. Er schränkte das Konzept aber wieder auf den Bereich der Historiographie ein. Für ihn bedeutete die Annahme des ,maurinischen Beispiels“ durch deutsche Benediktiner den „Abschied von der Tradition barocker Historiographie, von bloßer Kompilatorik, Apologetik, unkritischer Hagiographie, geistlichem und weltlichem Triumphalismus und vom Festhalten an ungeprüft adaptierten Autoritäten“" zugunsten systematischer Quellensammlung, kritischer Edition und analytisch-rekonstruierender Darstellung. Dass auch Neuerungen in der Wissenschaftsorganisation mit solchen Vorhaben einhergingen, hob Hammermayer als guter Kenner der Sozietäts- und Akademiebewegung ${ }^{7}$ hervor. Wo sich allerdings die ,wissenschaftliche Tätigkeit“ benediktinischer

Melk 39 (1889) 3-106, insb. 9-11, 26-29, 35-39, 44, 89-93. Die gründlichste Durchführung eines derartigen Ansatzes lieferte später Gall HEER, Johannes Mabillon und die Schweizer Benediktiner. Ein Beitrag zur Geschichte der historischen Quellenforschung im 17. und 18. Jahrhundert (St. Gallen 1938).

4 Franx Xaver Wegele, Geschichte der Deutschen Historiographie seit dem Auftreten des Humanismus (Geschichte der Wissenschaften in Deutschland. Neuere Zeit 20, München-Leipzig 1885) 547-549; zitiert bei MuSCHARD, Kirchenrecht (wie Anm. 1) 499. Wegele spricht aber nicht von „Maurinismus“, sondern nur allgemein von Entwicklung der „historischen Hilfswissenschaften“.

5 Wilhelm Wattenbach, Das Schriftwesen im Mittelalter (Leipzig 1871) 9-15, der auch Organisation und Zielsetzungen der Mauriner als Voraussetzungen für die Arbeit Mabillons würdigt; zitiert bei KaTSCHTHALER, Briefnachlass (wie Anm. 3) 9.

6 ENDRES, Korrespondenz (wie Anm. 3) 4.

7 Ludwig HAMMERMAYER, Marianus Brockie und Oliver Legipont - aus der benediktinischen Wissenschafts- und Akademiegeschichte des achtzehnten Jahrhunderts. Studien und Mitteilungen zur Geschichte des Benediktiner-Ordens und seiner Zweige 71 (1960) 69-121; Ludwig HAMMERMAYER, Zur Genese und Entfaltung von Aufklärung und Akademiebewegung im katholischen Oberdeutschland und zum Anteil des bayerischen Augustinerchorherrn-Stifts Polling (ca. 1717-1787), in: Europa in der Frühen Neuzeit. Festschrift für Günter MüHLPFORDT, 2: Frühmoderne, hg. von Erich DONNERT (Weimar-Köln-Wien 1997) 481-507. 
Gelehrter, wie bei Anselm Desing von Ensdorf ${ }^{8}$, auf zusätzliche Fachgebiete erweiterte, etwa Profangeschichte, Geschichtsdidaktik, Pädagogik, Kanonistik und Naturphilosophie, sah Hammermayer die Grenzen des „deutschen Maurinismus“ und zugleich die „Schwelle zur katholischen Frühaufklärung“ überschritten?.

Die Vorstellung eines maurinischen Einflusses auf süddeutsche Klöster wurde auch aus der Perspektive einer anderen Disziplin aufgegriffen und weiterentwickelt: der Kunstgeschichte. Christine Liebold erklärte in ihrer 1981 erschienenen Dissertation das bayerische Sakral-Rokoko zu einem ,von maurinischem Denken beeinflußten Stil““. „Bestimmte theologisch-wissenschaftliche Argumentationsweisen“ hätten den „Modus der kirchlichen Kunst $^{\text {" }}$ verändert ${ }^{10}$ - und zwar von einer auf affektiver Ebene wirkenden Suggestion der barocken Kunst in Richtung einer rationalen Demonstration der zu vermittelnden Inhalte. Die Thesen Liebolds sind im Einzelnen vielfach angefochten worden ${ }^{11}$, stellen allerdings die Verbindung her zwischen der Frage nach einem „Maurinismus“ und dem bedeutenden Forschungsstrang, der sich der Auseinandersetzung mit der Vergangenheit in der Kunst,

8 Ludwig Hammermayer, Anselm Desing, Abt von Ensdorf, in: Bayerische Kirchenfürsten, hg. von Ludwig SchrotT (München 1964) 238-247; Ildefons STEGMANN, Anselm Desing, Abt von Ensdorf 1699-1772. Ein Beitrag zur Geschichte der Aufklärung in Bayern (Studien und Mittheilungen zur Geschichte des Benediktiner-Ordens und seiner Zweige Ergh. 4, München 1929); Anselm Desing (16991772). Ein benediktinischer Universalgelehrter im Zeitalter der Aufklärung, hg. von Manfred KNEDLIK-Georg SCHROTT (Kallmünz 1999).

9 Ludwig Hammermayer, Zum „deutschen Maurinismus“ des frühen 18. Jahrhunderts. Briefe der Benediktiner P. Bernhard Pez (Melk) und P. Anselm Desing (Ensdorf) aus den Jahren 1709 bis 1725 . Zeitschrift für bayerische Landesgeschichte 40 (1977) 391-444, hier 392f.; vgl. Ludwig HAMmERMAYER, Die Forschungszentren der deutschen Benediktiner und ihre Vorhaben, in: Historische Forschung im 18. Jahrhundert: Organisation, Zielsetzung, Ergebnisse. 12. Deutsch-Französisches Historikerkolloquium des Deutschen Historischen Instituts Paris, hg. von Karl HAMMER-Jürgen Voss (Pariser Historische Studien 13, Bonn 1976) 122-191, hier $165-170$.

10 Christine LIEBOLD, Das Rokoko in ursprünglich mittelalterlichen Kirchen des bayerischen Gebietes - ein von maurinischem Denken geprägter Stil (Miscellanea Bavarica Monacensia. Dissertationen zur Bayerischen Landes- und Münchner Stadtgeschichte 98, München 1981) 29.

11 Am eingehendsten: Meinrad von Engelberg, Renovatio Ecclesiae. Die „Barockisierung“ mittelalterlicher Kirchen (Studien zur internationalen Architektur- und Kunstgeschichte 23, Petersberg 2005) 375-382. Tatsächlich sind die Ausführungen Liebolds zur Charakterisierung der Mauriner und ihres Einflusses ,auf die bayerischen Benediktiner und auf andere Orden in Bayern“ (LIEBOLD, Rokoko [wie Anm. 10] 2029) nicht nur knapp, sondern auch flüchtig gearbeitet und mit etlichen Tatsachenfehlern behaftet. 
insbesondere der sakralen Kunst, widmet ${ }^{12}$. Der Freisinger Dom und das Wirken Karl Meichelbecks sind dabei immer wieder ein bevorzugter Untersuchungsgegenstand gewesen ${ }^{13}$.

Die Kunst- und Architekturgeschichte kann auch, vor allem wenn sie neben dem Kirchen- auch den Konventbau einbezieht, dazu beitragen, die Verbindung zum Kontext der monastischen Lebensweise wiederherzustellen ${ }^{14}$. Es entspricht gewiss dem Selbstverständnis der maurinischen Benediktiner weit besser, sie nicht einseitig mit ihren Verdiensten um die Editionstechnik oder die Paläographie zu identifizieren, sondern gelehrte Betätigung - der im Übrigen ja auch nur eine winzige Minderheit der Mauriner nachging ${ }^{15}$ - als einen Baustein einer angestrebten umfassenden Erneuerung des monastischen Lebens einzuordnen ${ }^{16}$. Aus dieser Perspektive gelangt etwa Niklas Raggenbass, selbst Benediktiner in Engelberg, zur Rekonstruktion

12 Huberta WeIGL, Monastische Kunst und Geschichtsschreibung im 17. und 18. Jahrhundert. Zur Gegenwart der Vergangenheit, in: Mitteleuropäische Klöster der Barockzeit. Vergegenwärtigung monastischer Vergangenheit in Wort und Bild, hg. von Markwart Herzog-Huberta WeIGL (Irseer Schriften. Studien zur Wirtschafts-, Kultur- und Mentalitätsgeschichte N. F. 5, Konstanz 2011) 21-67.

13 Engelberg, Renovatio (wie Anm. 11) 249-261; Ulrike GöTz, Kunst in Freising unter Fürstbischof Johann Franz Eckher 1696-1727. Ausdrucksformen geistlicher Herrschaft (Sammelblatt des Historischen Vereins Freising 33, München-Zürich 1992) 147-190; Benno Hubensteiner, Die geistliche Stadt. Welt und Leben des Johann Franz Eckher von Kapfing und Liechteneck, Fürstbischofs von Freising (München 1954) 140-148, 177-192; vgl. in diesem Band den Beitrag von Uta Coburger.

14 Monique Bugner, Les bâtiments de la Congrégation de Saint-Maur, in: Sous la Règle de Saint Benoît. Structures monastiques et sociétés en France du moyen âge à l'époque moderne. Abbaye bénédictine Sainte-Marie de Paris, 23-25 Octobre 1980 (Hautes Études médiévales et modernes 47, Genève 1982) 539-554; Monique Bugner, Cadre architectural et vie monastique des bénédictins de la Congrégation de Saint-Maur (Nogent-le-Roi 1984); Bernard CHÉDOZEAU, Architecture et liturgie. La tridentinisation de l'abbatiale mauriste de Saint-Germain-des-Prés. Une église matricielle pour les églises mauristes?, in: Les Mauristes à Saint-Germain-des-Prés. Actes du Colloque de Paris (2 décembre 1999), hg. von Jean-Claude FredouilLE (Collection des Études Augustiniennes, Série Moyen Âge et Temps Modernes 36, Paris 2001) 27-46.

15 Einen Anteil von etwa $2 \%$ errechnet Pierre GASNAULT, Les travaux d'érudition des Mauristes au XVIIIe siècle, in: Historische Forschung im 18. Jahrhundert (wie Anm. 9) 102-121, hier 103f.

16 Manfred Weitlauff, Die Mauriner und ihr historisch-kritisches Werk, in: Historische Kritik in der Theologie. Beiträge zu ihrer Geschichte, hg. von Georg ScHWAIGER (Studien zur Theologie und Geistesgeschichte des Neunzehnten Jahrhunderts 32, Göttingen 1980) 153-209. Der Beitrag bleibt freilich der Selbstdarstellung der Mauriner in hohem Maße verhaftet und damit deutlich hinter dem Stand neuerer französischer Forschung, etwa: GaSnAult, Mauristes (wie Anm. 15); Madeleine 
eines „Reformprogramms der Mauriner“, das in deutschen Abteien nach dem Vorbild der französischen Kongregation angestrebt wurde ${ }^{17}$. Thomas Wallnig konnte jüngst sogar zeigen, dass manche Stränge gerade der frühen süddeutschen Rezeption des Wirkens der Mauriner ausschließlich auf deren asketisches Schrifttum ausgerichtet waren ${ }^{18}$ und sich mit dem Hammermayer'schen „Maurinismus“ im Sinne einer Ausbreitung der historischkritischen Methode weder inhaltlich noch personell überschnitten. Wenn man also von einem „maurinischen Modell“" sprechen will, dann sollte es jedenfalls weder auf den einzigen Aspekt der Gelehrsamkeit reduziert noch als „monolithisches Programm“ vorgestellt werden, sondern als „Bündel verschiedener administrativer, methodischer, religiöser und nicht zuletzt auch diskursiver Facetten“, welche in unterschiedlicher Auswahl und Intensität, bei entsprechend verschiedenen Resultaten, in einzelnen deutschen Klöstern rezipiert wurden ${ }^{19}$.

\title{
II. Karl Meichelbeck und die Mauriner
}

Die Beantwortung der im Titel gestellten Frage stößt mithin schon aufgrund der höchst unterschiedlichen möglichen Definitionen der Begriffe „Maurinismus" und „maurinisch“ auf ernste Schwierigkeiten. Der andere Terminus

\begin{abstract}
LAURAIN, Les travaux d'érudition des Mauristes: origine et évolution, in: Mémorial du XIV ${ }^{\mathrm{e}}$ centenaire de l'abbaye de Saint-Germain-des-Prés. Recueil de travaux sur le monastère et la congrégation de Saint-Maur (Bibliothèque de la Société d'Histoire Ecclésiastique de la France, Paris 1959) 231-271; Érudition et commerce épistolaire. Jean Mabillon et la tradition monastique, hg. von Daniel-Odon Hurel (Textes et traditions 6, Paris 2003); Dom Jean Mabillon, figure majeure de l'Europe des lettres. Actes des deux colloques du tricentenaire de la mort de dom Mabillon, Abbaye de Solesmes, 18-19 mai 2007, Palais de l'Institut, Paris, 7-8 décembre 2007, hg. von Jean LeClant-André VAuCheZ-Daniel-Odon Hurel (Paris 2010).

17 Niklas Raggenbass, „Harmonie und schwesterliche Einheit zwischen Bibel und Vernunft". Die Benediktiner des Klosters Banz: Publizisten und Wissenschaftler in der Aufklärungszeit (Studien und Mitteilungen zur Geschichte des Benediktinerordens und seiner Zweige Erg.bd. 44, St. Ottilien 2006) 16-88; Niklas RAGGENBASS, Reformprogramm der Benediktiner von St. Maur - Ideal und Realisierung im Kloster Banz. Studien und Mitteilungen zur Geschichte des Benediktinerordens und seiner Zweige 114 (2003) 175-248.
\end{abstract}

18 Thomas WALLNIG, La congrégation de Saint-Maur comme modèle d'ascèse. Exercices spirituels de Jérôme Le Contat et méditations de Claude Martin dans la traduction de Franz Mezger (Salzbourg), in: Dom Jean Mabillon (wie Anm. 16) 293-307.

19 Thomas Wallnig, Bernhard Pez und die Mauriner. Die Entstehung eines gelehrten Kontaktes im Spannungsfeld zwischen Vorbildhaftigkeit und Anregung, in: Érudition et commerce épistolaire (wie Anm. 16) 153-175, hier 153-155. 
der zu diskutierenden Proposition scheint leichter zu greifen: Persönlichkeit und Werk von Karl Meichelbeck, dem Benediktbeurer Benediktiner, Archivar und Historiker. An seinen Lebensweg, der gut erforscht ist ${ }^{20}$, sei hier nur knapp erinnert: 1669 in Marktoberdorf im Allgäu geboren, besuchte er die Klosterschule in Benediktbeuern, dann das Münchner Jesuitengymnasium ${ }^{21}$, legte 1688 in Benediktbeuern Profess ab und studierte erst am Kommunstudium der Bayerischen Benediktinerkongregation, dann an der Benediktineruniversität Salzburg ${ }^{22}$. Er unterrichtete auf Wunsch des Freisinger Bischofs Johann Franz Eckher von Kapfing am dortigen Gymnasium ${ }^{23}$, dann am

20 Franz Ludwig Baumann, Der bayerische Geschichtsschreiber Karl Meichelbeck 1669-1734. Festrede gehalten in der öffentlichen Sitzung der k. b. Akademie der Wissenschaften zu München zur Feier ihres 138. Stiftungstages am 27. März 1897 (München 1897); Stefan BeNZ, Zwischen Tradition und Kritik. Katholische Geschichtsschreibung im barocken Heiligen Römischen Reich (Historische Studien 473, Husum 2003) 602-611; Josef HemmerLe, Das Bistum Augsburg, 1: Die Benediktinerabtei Benediktbeuern (Germania Sacra N. F. 28, Die Bistümer der Kirchenprovinz Mainz, Berlin-New York 1991) 628-636; Pirmin LindNER, Fünf Professbücher süddeutscher Benediktiner-Abteien. Beiträge zu einem Monasticon-benedictinum Germaniae, 4: Professbuch der Benediktiner-Abtei Benediktbeuern (KemptenMünchen 1910) 61-73, 152-164; ergänzend: Laurentius KосH, Der Typus des „monachus eruditus historicus“ der Barockzeit und der Frühaufklärung im süddeutsch-katholischen Raum am Beispiel des Benediktiner-Historikers P. Carl Meichlbeck, in: Literaten - Kleriker - Gelehrte. Zur Geschichte der Gebildeten im vormodernen Europa, hg. von Rudolf W. KECK-Erhard WIERSING-Klaus WiTTSTADT (Beiträge zur historischen Bildungsforschung 15, Köln-Weimar-Wien 1996) 289302; Leo WeBER, Karl Meichelbeck OSB (1669-1734). Jahrbuch des Vereins für Augsburger Bistumsgeschichte 39 (2005) 129-145.

21 Maximilian LeITsCHuH, Die Matrikeln der Oberklassen des Wilhelmsgymnasiums in München (Schriften des Wilhelmsgymnasiums in München, 4 Bde., München 1970-1976) 2 35; Maximilian LeITSCHUH, Die Schüler des Wilhelmsgymnasiums, in: Festschrift zur Vierhundert-Jahr-Feier des Wilhelms-Gymnasiums 1559-1959, hg. von Siegfried HÄFNER (München 1959) 9-37, hier 20.

22 Karl MinderA, Die Jugend Karl Meichelbecks und sein Weg zur Geschichtsforschung. Studien und Mitteilungen zur Geschichte des Benediktiner-Ordens und seiner Zweige 80/1-2 (1969) 61-104; zum Studium in Salzburg: Virgil RedLiCH, Die Matrikel der Universität Salzburg 1639-1810, 1: Text der Matrikel (Salzburger Abhandlungen und Texte aus Wissenschaft und Kunst 5, Salzburg 1933) 198. Zu den Lehrinhalten der Salzburger Universität: Emmanuel J. BAUER, Thomistische Metaphysik an der alten Benediktineruniversität Salzburg. Darstellung und Interpretation einer philosophischen Schule des 17./18. Jahrhunderts (Salzburger Theologische Studien 1, Innsbruck-Wien 1996).

23 Martin von Deutinger, Zur Geschichte des Schulwesens in der Stadt Freising. Beyträge zur Geschichte, Topographie und Statistik des Erzbisthums München und Freising 5 (1854) 209-568, hier 453; vgl. HubENSTEINER, Geistliche Stadt (wie Anm. 13) $177 \mathrm{f}$. 
Kommunstudium $^{24}$. In seinem Kloster war er Bibliothekar, Kustos der Anastasia-Reliquien, vor allem aber viele Jahre Archivar ${ }^{25}$. Die Bayerische Benediktinerkongregation betraute ihn mit der Führung ihrer Kongregationsannalen $^{26}$. Etwas über fünf Jahre lebte er in Freising, wo er im Auftrag Eckhers für die Millenniumsfeier von 1724 die Geschichte des Bistums bearbeitete $^{27}$. Am 2. April 1734 starb Meichelbeck in Benediktbeuern.

In Erinnerung geblieben ist er vor allem als Verfasser der zwischen 1724 und 1729 in vier Bänden erschienenen Historia Frisingensis ${ }^{28}$ sowie des posthum veröffentlichten Chronicon Benedictoburanum ${ }^{29}$. Insbesondere die erstere Arbeit, seine Geschichte des Bistums Freising unter ausgiebiger Benutzung handschriftlicher Quellen, fand bereits unmittelbar nach ihrem Erscheinen breite Anerkennung beim gelehrten Publikum ${ }^{30}$ und gilt bis heute vielfach als das erste Geschichtswerk aus Süddeutschland, das „die Anforderungen der kritischen Methode erfüllt $[\mathrm{e}]^{\text {(31 }}$ beziehungsweise ,mau-

24 Anselm REICHHOLD, 300 Jahre Bayerische Benediktiner-Kongregation im Spiegel der wichtigsten Beschlüsse der Generalkapitel. Studien und Mitteilungen zur Geschichte des Benediktiner-Ordens und seiner Zweige 95 (1984) 522-696, hier 672. Zur Institution: Stephan HAERING, Studien und Wissenschaft in der Bayerischen Benediktinerkongregation vor 1803. Ein Überblick. Studien und Mitteilungen zur Geschichte des Benediktiner-Ordens und seiner Zweige 101 (1990) 121-138, hier 124-129.

25 Karl Mindera, P. Karl Meichelbecks Benediktbeurer Archiv. Mitteilungen für die Archivpflege in Bayern 13 (1967) 35-46.

26 Albert SiEgmund, Die Annales Congregationis Benedictino-Bavaricae (16841772). Studien und Mitteilungen zur Geschichte des Benediktiner-Ordens und seiner Zweige 78 (1967) 144-167, hier 153-158.

27 Baumann, Meichelbeck (wie Anm. 20) 16-18; Götz, Kunst in Freising (wie Anm. 13) 246-249, 260, 272-274; HubensteIner, Geistliche Stadt (wie Anm. 13) 181-189; Wilhelm WÜHR, Meichelbecks Bedeutung für die deutsche Geschichtsschreibung. 225 Jahre „Historia Frisingensis“, in: Festschrift Kardinal FAULHABER zum achtzigsten Geburtstag dargebracht vom Professorenkollegium der Philosophisch-theologischen Hochschule Freising (München 1949) 219-239, hier 232-237.

28 Karl Meichelbeck, Historia Frisingensis (4 Bde., Augsburg-Graz 1724-1729).

29 Karl Meichelbeck, Chronicon Benedictoburanum, in quo ex incunabulis, vicissitudinibus, decrementis, incrementis monasterii, actis abbatum et aliorum virorum celebrium, historia Germaniae a saeculo Christi VIII. usque ad saeculum XVIII. [...] elucidatur, hg. von Alphons HAIDENFELD (2 Bde., München 1751).

30 Meichelbeck selbst freute sich besonders über die durch Gottfried Bessel vermittelte günstige Aufnahme am Kaiserhof, jene an der päpstlichen Kurie sowie die Rezension in den Leipziger Acta eruditorum: Hauptstaatsarchiv (HStA) München, Klosterliteralien (KL) Benediktbeuern 125 II, 72r-73r, 79r-v; Aegidius KoLB, Karl Meichelbecks Autobiographie. Das eigenhändig verfaßte Compendium Gestorum. Studien und Mitteilungen zur Geschichte des Benediktiner-Ordens und seiner Zweige 80/1-2 (1969) 41-60, hier 57.

31 BAUMANn, Meichelbeck (wie Anm. 20) 11; ähnlich WÜHr, Bedeutung (wie Anm. 27) 219. 
rinischen Anforderungen im wesentlichen entsprach“332. Die „Historia Frisingensis" ist damit auch das wohl zentrale Beweisstück dafür, Meichelbeck als „Protagonist[en] im Sinne der Mauriner“33 oder mit Hammermayer als „führenden Repräsentanten“ des deutschen Maurinismus neben Bernhard Pez einzustufen ${ }^{34}$. Er zählt im Übrigen auch - zusammen mit Gottfried Bessel $^{35}$, Martin Gerbert ${ }^{36}$ und eben Bernhard Pez - zu jener Handvoll süddeutscher Benediktiner, denen das epitheton ornans eines „deutschen Mabillon" beigelegt worden ist ${ }^{37}$. Wäre dieser Ausdruck nicht nachweislich viel

32 HAMmermayer, Forschungszentren (wie Anm. 9) 145; ähnlich Hammermayer, Maurinismus (wie Anm. 9) 404; Hubensteiner, Geistliche Stadt (wie Anm. 13) 192.

33 LieBOLD, Rokoko (wie Anm. 10) 24.

34 Hammermayer, Maurinismus (wie Anm. 9) 400.

35 Emmeram RitTeR, Gottfried Bessel - der „,deutsche Mabillon“, in: Gottfried Bessel (1672-1749). Diplomat in Kurmainz - Abt von Göttweig - Wissenschaftler und Kunstmäzen, hg. von Franz Rudolf REICHERT (Quellen und Abhandlungen zur mittelrheinischen Kirchengeschichte 16, Mainz 1972) 203-215; Peter G. TropPER, Abt Gottfried Bessel (1714-1749), in: 900 Jahre Stift Göttweig 1083-1983. Ein Donaustift als Repräsentant benediktinischer Kultur. Stift Göttweig Jubiläumsausstellung, Stift Göttweig, 29. April bis 26. Oktober 1983, im Kaiser- und Fürstentrakt mit Prälatur, hg. von Gregor Martin LECHNER (Furth bei Göttweig-Wien 1983) 644-686; Edmund VAŠIČEK, Abt Gottfried Bessel von Göttweig. Ein Lebensbild (Studien und Mitteilungen aus dem kirchengeschichtlichen Seminar der theologischen Fakultät der k. k. Universität in Wien 10, Wien 1912).

36 Alfons DeIssler, Fürstabt Martin Gerbert von St. Blasien und die theologische Methode. Eine Studie zur deutschen Theologiegeschichte des 18. Jahrhunderts (Studien und Mitteilungen zur Geschichte des Benediktiner-Ordens und seiner Zweige Ergh. 15, München 1940); Franz M. HiLger, Martin Gerbert. Fürst und Abt von St. Blasien. Festschrift zur 250. Wiederkehr seines Geburtstages (Konstanz 1970); Wolfgang MüLLER, Martin Gerbert. Abt - Landesherr - Wissenschaftler, in: St. Blasien. Festschrift aus Anlaß des 200jährigen Bestehens der Klosterund Pfarrkirche, hg. von Heinrich HeIDEgGER-Hugo OTт (München-Zürich 1983) 127-133; Georg Pfeilschifter, Korrespondenz des Fürstabtes Martin II. Gerbert von St. Blasien, 1: 1752-73 (Karlsruhe 1931) VII-XII.

37 Belege aus dem 18. Jh. zu Bessel und Gerbert bringt HAMMERMAYER, Forschungszentren (wie Anm. 9) 165. Pez wurde bereits 1717 von Johann Albert Fabricius als novus quidam [...] hodie Mabillonius bezeichnet: Joannis Mabillonii Iter Germanicum et Joannis Launoii De scholis celebribus a Carolo Magno et post Carolum Magnum in Occidente instauratis liber, hg. von Johann Albert FABRICIUS (Hamburg 1717), Ad lectorem (unpag.); als „der deutsche Mabillon“ erscheint er bei ENDRES, Korrespondenz (wie Anm. 3) 9. Für Meichelbeck findet sich „bayerischer Mabillon“ anscheinend erst im 20. Jh., so bei HEMMERLE, Benediktbeuern (wie Anm. 20) 276; Maximilian Joseph HufNAGEL, Das 1500-jährige Maximilians-Jubiläum im Hochstift Freising vom Jahre 1772. Pflege frühmittelalterlicher Tradition im Zeitalter des Spätbarock, in: Bavaria christiana. Zur Frühgeschichte des Christentums in Bayern. Fest- 
älter als ,deutscher Mauriner“, man wäre versucht, ihn als Superlativ desselben anzusehen.

Zugleich gilt allerdings eine nähere Ausleuchtung der Verbindungen Meichelbecks zu seinen vermuteten Vorbildern in Frankreich den Autoren, die diese Urteile abgegeben haben, häufig weiterhin als Desiderat ${ }^{38}$. Es scheint somit nicht müßig, das bereits geschlossene Dossier wieder aufzumachen und die titelgebende Frage nochmals zu stellen - wobei das bisher Dargelegte sie zu präzisieren hilft. Die Frage, ob Meichelbeck ein „deutscher Mauriner" war, bedarf einer zweifachen Ausdehnung: Aufgrund der festgestellten definitorischen Unschärfe wäre zu differenzieren, inwiefern er einer war, und aufgrund der konstatierten Lücke in einem sonst - gerade im Vergleich zu anderen klösterlichen Gelehrten - günstigen biographischen Forschungsstand ist zu fragen, wie und wann er einer wurde. Gerade diese letztere Frage hat freilich schon frühere Generationen von Biographen frustriert, und es sei vorausgeschickt, dass auch dieser Beitrag ihre endgültige Beantwortung nicht leisten kann.

Dass sich manches Neue doch vorbringen lässt, ist nicht zuletzt der erfreulichen Quellenlage zu Meichelbeck geschuldet. Als Grundlagen für eine Analyse seines Verhältnisses zu den Maurinern und ihren Werken stehen einerseits seine gedruckten Arbeiten zur Verfügung, andererseits sein großteils in der Bayerischen Staatsbibliothek verwahrter handschriftlicher Nachlass. Dieser umfasst neben einigen ungedruckt gebliebenen Schriften die Konzepte und Druckvorlagen seiner publizierten Werke sowie kostbare autobiographische Aufzeichnungen. Hierzu zählen das knappe Compendium gestorum oder Nucleus memorabilium ${ }^{39}$, aber auch viele, teils umfängliche Berichte über seine Aktivitäten, die er den Kongregationsannalen einfügte ${ }^{40}$.

schrift Adolf Wilhelm ZIEGLER, hg. von Wilhelm GeSSEL-Peter STOCKMEIER (Beiträge zur altbayerischen Kirchengeschichte 27, München 1973) 205-224, hier 210; MinderA, Benediktbeurer Archiv (wie Anm. 25) 35; RedLICH, Matrikel Salzburg (wie Anm. 22) V; Laura ScHERR, Studien zur Geschichte des Augustiner Chorherrenstiftes Ranshofen am Inn und seines Archivs. Mitteilungen des Oberösterreichischen Landesarchivs 21 (2008) 143-247, hier 199. Als „,deutschen Mabillon“ bezeichnet ihn Georg SCHWAIGER, Kirche und Kultur im barocken Bayern. Zum 300. Geburtstag P. Karl Meichelbecks OSB. Studien und Mitteilungen zur Geschichte des Benediktiner-Ordens und seiner Zweige 80/1-2 (1969) 7-20, hier 20.

38 Baumann, Meichelbeck (wie Anm. 20) 32f.; HAMmermayer, Forschungszentren (wie Anm. 9) 142-144.

39 Bayerische Staatsbibliothek (BStB) München, Meichelbeckiana 19; ediert von KoLB, Autobiographie (wie Anm. 30); vgl. Karl DACHS, Die Meichelbeckiana der Bayerischen Staatsbibliothek. Studien und Mitteilungen zur Geschichte des BenediktinerOrdens und seiner Zweige 80/1-2 (1969) 190-204, hier 200.

40 HStA München, KL Benediktbeuern 125 I-II; vgl. DACHS, Meichelbeckiana (wie Anm. 39) 190; Siegmund, Annales (wie Anm. 26) 163f. 
Vor allem zu nennen sind aber die mehr als vierzig Jahre hindurch, von 1692 bis 1734, kontinuierlich geführten Diarien Meichelbecks ${ }^{41}$. Neben ihrer Ausführlichkeit sind sie auch durch die Unverblümtheit wertvoll, mit welcher der Verfasser seinen Gefühlen oftmals freien Lauf lässt; die teils herbe Kritik an Haltungen seines Abtes und seiner Mitbrüder zeigt, dass er großes Vertrauen in die Unverletzlichkeit der Aufzeichnungen hatte, deren primärer Adressat wohl er selbst war ${ }^{42}$. Schließlich kommen noch Briefe Meichelbecks als Quellen in Frage, von denen etwas mehr als 100 erhalten sind ${ }^{43}$.

Von den Möglichkeiten, mit der Maurinerkongregation Kontakt zu pflegen und auf einen Transfer von Kenntnissen und Lebensweise hinzuarbeiten, bestand die intensivste darin, dass deutsche Benediktiner einige Zeit in St.-Germain lebten und dort studierten. Pläne in dieser Richtung waren bereits nach der Deutschlandreise Mabillons von 1683 ventiliert worden ${ }^{44}$, ernst wurde es damit aber erst um 1720. Aus St. Emmeram zu Regensburg wurde Johann Baptist Kraus 1721 zu einem etwa zweijährigen Aufenthalt entsandt $^{45}$, wenig später aus St. Blasien Marquard Herrgott ${ }^{46}$. Bernhard Pez,

41 BStB München, Meichelbeckiana 18a-18c; vgl. DACHS, Meichelbeckiana (wie Anm. 39) 199; MinderA, Jugend (wie Anm. 22) 70.

42 Hierfür spricht nicht zuletzt, dass Meichelbeck in den frühesten Eintragungen von sich selbst in der zweiten Person Singular spricht; vgl. HubENSTEInER, Geistliche Stadt (wie Anm. 13) 178; MinderA, Jugend (wie Anm. 22) 70; WÜHR, Bedeutung (wie Anm. 27) 226. Ab etwa 1697 ging er zur ersten Person über.

43 Obwohl unvollständig geblieben, ist die Edition dieser Briefe höchst wertvoll: Albert Siegmund, P. Karl Meichelbecks Briefe. Studien und Mitteilungen zur Geschichte des Benediktiner-Ordens und seiner Zweige 80/1-2 (1969) 105-189; 81 (1970) 261314. Eine Übersicht der erhaltenen Stücke ebd. 80/1-2 107-115.

44 Eine Anfrage des Abtes Benedikt Hornstein von Ottobeuren an Mabillon zu einem möglichen Aufenthalt seines Konventualen Sebastian Textor findet sich in: Bibliothèque nationale de France (BNF), Fonds français 19679, 134r-v (undatiert, zwischen 1683 und 1688). Zu der Reise kam es wohl nicht; nichts wissen davon Pirmin LINDNER, Album Ottoburanum. Die Äbte und Mönche des ehemaligen freien ReichsStiftes Ottobeuren, Benediktiner-Ordens in Schwaben und deren literarischer Nachlass von 764 bis zu ihrem Aussterben (1858). Zeitschrift des Historischen Vereins für Schwaben und Neuburg 30 (1903) 77-142; 31 (1904) 1-90, hier 30 141f.; Walter STEINBÖCK, P. Sebastian Textor. Das Leben und Wirken des Ottobeurer Mönches und Salzburger Universitätsprofessors zur Zeit des Fürsterzbischofs Ernst Thun. Versuch einer Biographie. Ein Beitrag zur Geschichte der Salzburger BenediktinerUniversität. Studien und Mitteilungen zur Geschichte des Benediktiner-Ordens und seiner Zweige 83 (1972) 755-834, hier 787f.

45 Endres, Korrespondenz (wie Anm. 3) 17-27; Egon Johannes GreIPL, Abt und Fürst. Leben und Leistung des Reichsprälaten Johann Baptist Kraus von St. Emmeram zu Regensburg (1700-1762) (Regensburg 1980) 38-40.

46 Josef Peter ORTNER, Marquard Herrgott (1694-1762). Sein Leben und Wirken als Historiker und Diplomat (Veröffentlichungen der Kommission für Geschichte Öster- 
der eine solche Studienreise schon seit mehr als zehn Jahren anstrebte, konnte sie nach vielfältigen Widerständen seines Abtes 1728 endlich antreten ${ }^{47}$. Dass Meichelbeck, der um diese Zeit bereits das fünfzigste Lebensjahr hinter sich hatte, nie nach Paris ging, ist nicht allzu erstaunlich; es sei jedoch festgehalten, dass auch an die Entsendung eines jüngeren Benediktbeurers nicht gedacht worden zu sein scheint. Jedenfalls findet sich darauf in den Quellen nirgends ein Hinweis. Freilich waren die Hürden für eine solche Reise hoch - nicht nur finanziell, denn der Aufenthalt in Paris war teuer ${ }^{48}$, sondern auch politisch und institutionell. Das Reichsstift St. Blasien hatte es hier nicht nur der bloßen Entfernung halber leichter als das bayerisch-landständische Kloster Benediktbeuern.

Die wichtigste Form direkter Verbindung mit St.-Germain war jedoch Korrespondenz. Die Maurinergelehrten kannten den Wert brieflicher Kommunikationsnetze als Nervengeflecht der res publica literaria zur Genüge und waren sehr bemüht um die Pflege von commercia literaria auch über nationale und konfessionelle Grenzen hinweg ${ }^{49}$; gerade benediktinischen Mitbrüdern aus dem Ausland gaben sie meist bereitwillig Antwort. Bernhard Pez gelang die Anknüpfung einer Verbindung noch 1709, als der Spanische Erbfolgekrieg in vollem Gange war, über die Vermittlung St. Gallens ${ }^{50}$.

reichs 5, Wien 1972) 18-24; vgl. BADER, Sanct Blasien (wie Anm. 3) 177f.; DeISSLER, Gerbert (wie Anm. 36) 39.

47 Katschthaler, Briefnachlass (wie Anm. 3) 89-93; HAMmermayer, Maurinismus (wie Anm. 9) 407; Wallnig, Pez und Mauriner (wie Anm. 19) 161.

481714 wurden die jährlichen Kosten mit 500 Livres beziffert: Thomas WallNiGThomas Stockinger, Die gelehrte Korrespondenz der Brüder Pez. Text, Regesten, Kommentare, 1: 1709-1715 (Quelleneditionen des Instituts für Österreichische Geschichtsforschung 2/1, Wien-München 2010) 599-602 Nr. 364 (René Massuet an Bernhard Pez, 5. Oktober 1714); vgl. LAURAIN, Travaux d'érudition (wie Anm. 16) 246f.

49 Daniel-Odon Hurel, La correspondance des Bénédictins de la Congrégation de SaintMaur $\left(\mathrm{XVII}^{\mathrm{e}}-\mathrm{XVIII}^{\mathrm{e}}\right.$ siècles): corpus et pratiques de sociabilité, in: Correspondance et sociabilité, hg. von Daniel-Odon Hurel (Publications de l'Université de Rouen 201, Rouen 1994) 59-66; Daniel-Odon HuREL, Correspondance épistolaire et vie monastique chez les bénédictins de la Congrégation de Saint-Maur (XVII ${ }^{\mathrm{e}}$ XVIII ${ }^{\mathrm{e}}$ siècles). Recherches Augustiniennes 27 (1994) 187-212.

50 Wallnig-Stockinger, Korrespondenz 1 (wie Anm. 48) 74-76 Nr. 24, 81-85 Nr. 32, 117-122 Nr. 48; vgl. Gall HeER, P. Bernhard Pez von Melk OSB (1683-1735) in seinen Beziehungen zu den Schweizer Klöstern. Ein Kapitel benediktinischer Geistesgeschichte des frühen 18. Jahrhunderts, in: Festschrift Oskar VASELLA. Zum 60. Geburtstag am 15. Mai 1964 überreicht von Schülern und Freunden (Freiburg 1964) 403-455, hier 403-405; Katschthaler, Briefnachlass (wie Anm. 3) 23; Thomas StOCKINGER, „Fidelis tametsi inutilis servus“. P. Moritz Müller OSB (St. Gallen) in seiner historisch-literarischen und politisch-diplomatischen Tätigkeit im Spiegel sei- 
Kaspar Erhardt ${ }^{51}$ in St. Emmeram stellte spätestens 1715 einen ersten Kontakt mit Pez' Briefpartner René Massuet ${ }^{52}$ her. Aus dem Zeitraum bis 1738 sind insgesamt 43 Schreiben von zehn verschiedenen Maurinern nach St. Emmeram überliefert, die Korrespondenz setzte sich bis ins späte 18. Jahrhundert fort ${ }^{53}$. Auch mehrere schwäbische Benediktinergelehrte standen in regelmäßigem Kontakt mit den Parisienses oder Galli nostri, wie man die französischen Ordensbrüder oft nannte ${ }^{54}$.

Dass auch Meichelbeck ein Korrespondent der Mauriner gewesen sei, ist der Literatur an einigen Stellen zu entnehmen ${ }^{55}$. Die erhaltenen Bestände der Maurinerkorrespondenz in der Pariser Bibliothèque nationale ${ }^{56}$ enthalten jedoch keinen Brief von ihm; freilich ist diese Sammlung zwar umfangreich, aber keineswegs vollständig. Die von Meichelbeck erhaltenen Briefe, die er selbst akribisch sammelte, sind heute nahezu vollständig verloren ${ }^{57}$. In

ner Korrespondenz 1709-1714. Studien und Mitteilungen zur Geschichte des Benediktinerordens und seiner Zweige 118 (2007) 339-432, hier 348-355.

51 ENDRES, Korrespondenz (wie Anm. 3) 9-26; Friedrich LAUCHERT, Erhard: Kaspar, in: Allgemeine Deutsche Biographie, 48 (Leipzig 1904) 392-393; WALLNIGSTOCKINGER, Korrespondenz 1 (wie Anm. 48) 800.

52 René-Prosper TAssin, Histoire littéraire de la congrégation de Saint-Maur, ordre de S. Benoît (Bruxelles 1770) 375-379; vgl. CHAussy, Bénédictins (wie Anm. 2) 2 58; Yves Chaussy, Matricula Monachorum Professorum Congregationis S. Mauri in Gallia Ordinis Sancti Patris Benedicti. Ab initio eiusdem Congregationis, usque ad annum 1789 (Bibliothèque d'histoire et d'archéologie chrétiennes, Paris 1959) 71; Jean-Baptiste VANEL, Les Bénédictins de Saint-Germain-des-Prés et les savants lyonnais d'après leur correspondance inédite (Paris-Lyon 1894) 289-375.

53 Eine Übersicht findet sich bei ENDRES, Korrespondenz (wie Anm. 3) $5 f$.

54 Beispielsweise Anselm Fischer in Ochsenhausen oder Felix Egger in Petershausen: WALlNiG-Stockinger, Korrespondenz 1 (wie Anm. 48) 110-113 Nr. 43, 181f. Nr. 94, 355f. Nr. 219, 391-394 Nr. 246, 394-400 Nr. 248, 465-467 Nr. 281; Briefe Eggers in: BNF, Fonds français 17706, 156r-157v; 19652, 194r-195v; 19662, 98rv; 19664, 119r-128v; Fischer: ebd. Fonds français 19664, 138r-139v; 19665, $310 \mathrm{r}-313 \mathrm{v}$.

55 Endres, Korrespondenz (wie Anm. 3) 10f.; LiEBold, Rokoko (wie Anm. 10) 25, 124; Richard VAN DÜLMEN, Anfänge einer geistigen Neuorientierung in Bayern zu Beginn des 18. Jahrhunderts. Eusebius Amorts Briefwechsel mit Pierre-François Le Courayer in Paris. Zeitschrift für bayerische Landesgeschichte 26 (1963) 493-559, hier 495, 499. Alle beruhen auf dem in Anm. 60 angegebenen Beleg Baumanns.

56 BNF, Fonds français 12803-12804, 17667, 17678-17713, 19639-19681, 20941, 22313, 25537-25538; BNF, Fonds latin 11645, 11656-11657, 11662, 11664, 11666, 12658-12703, 12706; vgl. Pierre GaSNAUlt, La correspondance des Mauristes aux $\mathrm{XVII}^{\mathrm{e}}$ et XVIII ${ }^{\mathrm{e}}$ siècles, in: Sous la Règle de Saint Benoît (wie Anm. 14) 293-304, hier $293 f$.

57 Siegmund, Meichelbecks Briefe (wie Anm. 43) 80/1-2 104f.; vgl. Lindner, Professbuch Benediktbeuern (wie Anm. 20) 65. In HStA München, KL Fasz. 102/17a, sind einige Originalbriefe an Meichelbeck unter anderem von dem Hofkammer- 
seinem Diarium allerdings pflegte Meichelbeck erhaltene wie versandte Briefe zu verzeichnen, auch dies zwar nicht lückenlos, aber doch in überwiegender Zahl. Festzustellen ist nun, dass die vollständige Durchsicht dieser Diarien nicht eine einzige Erwähnung eines Briefwechsels mit einem Mauriner oder überhaupt mit irgendeinem Korrespondenten in Frankreich ergibt.

Den einzigen bisher bekannten Beleg für einen solchen Kontakt liefert ein Auszug aus einem Brief von Pierre Thibault ${ }^{58}$, den Meichelbeck seinen Kongregationsannalen zum Jahr 1725 einfügte. Eingeleitet wird das Zitat mit den Worten: „Um etwa dieselbe Zeit kam von Paris aus dem Kloster St.-Germain-des-Prés ein Antwortbrief des hochwürdigen Paters Pierre Thibault an uns, welcher von einem der unseren über die in der Kongregation des Hl. Maurus gewöhnlich gehaltene Studienordnung befragt worden war ${ }^{\text {‘59. }}$. Franz Ludwig Baumann, der als Erster auf diese Passage hinwies, deutete quidam ex nostris als Umschreibung für Meichelbeck selbst, der sich auch an weiteren Stellen auf diese Art verhüllt ${ }^{60}-$ an vielen anderen jedoch namentlich von sich selbst spricht. Auch hier bietet das Diarium während des gesamten fraglichen Jahres keinerlei Hinweis, dass Meichelbeck eine Anfrage nach Paris geschrieben oder eine Antwort Thibaults erhalten hätte ${ }^{61}$. Dass er gerade einen so außergewöhnlichen Brief nicht erwähnen sollte, wäre erstaunlich und gegen seine sonstige Gewohnheit. Es ist daher in Betracht zu ziehen, dass Baumann geirrt haben und der Korrespondent Thibaults doch ein anderer bayerischer Benediktiner gewesen sein könnte.

Hierzu ließe sich etwa die folgende Konjektur aufstellen, für die freilich ein substantieller Quellenbeleg ebenso fehlt wie für Meichelbeck als

beamten Karl Peter in Innsbruck und dem Schneider Georg Karl in Rom erhalten; keines dieser Stücke betrifft gelehrte Themen.

58 Prior von St.-Germain-des-Prés 1720-1725, Generalsuperior der Mauriner 17251729: Chaussy, Bénédictins (wie Anm. 2) 1 139-157; 2 52; Chaussy, Matricula (wie Anm. 52) 65; Jean-Baptiste Vanel, Les Bénédictins de Saint-Maur à SaintGermain-des-Prés 1630-1792. Nécrologe des religieux de la Congrégation de Saint-Maur décédés à l'Abbaye de Saint-Germain-des-Prés (Paris 1896) 341, 348.

59 HStA München, KL Benediktbeuern 125 I, 80v: Eodem fere tempore venere Parisiis ex monasterio $S$. Germani a Pratis ad nos literae responsoriae reverendi patris Petri Thibaudt, qui a quodam ex nostris interrogatus fuerat super ratione studiorum in congregatione sancti Mauri observari solita.

60 Baumann, Meichelbeck (wie Anm. 20) 48; vgl. Stegmann, Desing (wie Anm. 8) 232f. - VAN Dülmen, Anfänge (wie Anm. 55) 495, zitiert aus derselben Passage der Annalen unter der irrigen Behauptung, es handle sich um einen Brief Meichelbecks an den Mauriner Vincent Thuillier.

61 BStB München, Meichelbeckiana 18c, 134v-165v. 
Empfänger des Briefes von Thibault. Im Herbst 1725 kam der junge Anselm Desing als neuer Professor der Rudimenta ans Freisinger Gymnasium ${ }^{62}$, wo er vielfachen Kontakt mit Meichelbeck hatte ${ }^{63}$ und diesem später auch bei den Arbeiten am zweiten Band der Historia Frisingensis zur Hand ging ${ }^{64}$. Zugleich leistete er in seiner Freisinger Zeit große Beiträge zur Umstellung der Lehrmethoden des Gymnasiums unter Forcierung des Geschichtsunterrichts ${ }^{65}$. Dass er dabei auf die Mauriner und besonders auf Mabillons Traité des études monastiques ${ }^{66}$ rekurrierte, ist gut nachweisbar. Bereits in seinem Erstlingswerk Methodus contracta historiae, das er 1725 kurz vor dem Gang nach Freising veröffentlichte, findet sich eine Würdigung der historischen Leistungen der Mauriner unter explizitem Verweis auf den Traité, freilich in Kombination mit Darlegungen zu Wesen und Inhalt der Geschichte, die noch zutiefst von der scholastischen Philosophie geprägt $\operatorname{sind}^{67}$. In seinem universalgeschichtlichen Abriss Compendium eruditionis von 1728 findet sich ein ausführlicheres Elogium Mabillons, den ,die neueste Zeit als Fürsten der Gelehrsamkeit" verehre ${ }^{68}$; auch in einem Brief von 1731 empfahl Desing den Traité zur Unterweisung des benediktinischen Nachwuchses ${ }^{69}$. Somit erscheint es zumindest plausibel, dass er um 1725 an einer Kontaktaufnahme mit den Maurinern in Fragen der Studienordnung interessiert gewesen sein könnte. Er stand nachweislich in Verbindung mit den Gelehrten von St. Emmeram, zu deren Korrespondenten unter den Maurinern Thibault zählte $^{70}$. Über die Emmeramer, Desing oder beide könnte der Brief an Meichelbeck und in die Annales gelangt sein. Falls dies zutrifft, dann wären alle

62 Stegmann, Desing (wie Anm. 8) $20 \mathrm{f}$.

63 BStB München, Meichelbeckiana 18c, 162r (28. 10. 1725), 164v (16. 12. 1725).

64 Stegmann, Desing (wie Anm. 8) 27.

65 Stegmann, Desing (wie Anm. 8) 22f.

66 Jean Mabillon, Traité des études monastiques, divisé en trois parties (Paris 1691); Jean Mabillon, Tractatus de studiis monasticis divisus in tres partes, übers. von Ulrich STAUdigL (Kempten 1702).

67 Anselm Desing, Methodus contracta historiae (Amberg 1725) 1 16r-17r; vgl. Stegmann, Desing (wie Anm. 8) 156-159.

68 Anselm Desing, Compendium eruditionis (München 1728) 222: [...] quem aetas proxime lapsa eruditionis principem adorat; zu diesem Werk vgl. Guillaume vAN GEMERT, Katholische Bildung an der Schwelle zur Aufklärung zwischen Tradition und Neuorientierung. Anselm Desings Compendium Eruditionis (1728) als pädagogische Programmschrift, in: Anselm Desing (wie Anm. 8) 164-184.

69 Johannes SCHABER, Zwischen Barockscholastik und Kirchenväterrenaissance. Anselm Desing im Wandel benediktinischer Studienreformen des 18. Jahrhunderts, in: Anselm Desing (wie Anm. 8) 106-131, hier 121.

70 Hammermayer, Desing (wie Anm. 8) 241; Hammermayer, Maurinismus (wie Anm. 9) 414f. Erhalten sind vier Briefe Thibaults nach St. Emmeram: ENDRES, Korrespondenz (wie Anm. 3) 73-76. 
Angaben der Literatur über Meichelbecks Maurinerkorrespondenz hinfällig, denn bei näherer Betrachtung gehen sie durchwegs auf Baumann zurück wenn sie dessen Angabe auch teils überinterpretieren, bis zur haltlosen Behauptung, für Meichelbeck lasse sich „von 1715 an [...] ein ständiger Briefwechsel mit den Maurinern nachweisen “"71.

Selbst wenn dieser Beleg auszuscheiden ist, beweist das sonstige Schweigen der Quellen nicht zweifelsfrei, dass der Benediktbeurer nie einen Brief nach St.-Germain geschrieben oder von dort erhalten habe. Festgehalten sei hier allerdings, dass er regelmäßige Korrespondenz mit maurinischen Gelehrten, die mit jener der Emmeramer oder Melker zu vergleichen wäre, nicht geführt hat.

Eine verwandte Frage ist die der Wissenschaftsorganisation und des höheren Bildungswesens. Die Bedeutung der Prälatenorden, speziell der Benediktiner und Augustiner-Chorherren, für die Akademiebewegung in Süddeutschland ist von Hammermayer, Andreas Kraus und Alois Schmid in zahlreichen Publikationen herausgestrichen worden ${ }^{72}$. Es ist auch unbestreitbar, dass das Vorbild der Mauriner einige dieser Bemühungen inspirierte: bei Pez, dessen Wiener Akademievorschläge in einer ersten Phase von dem jungen Johann Christoph Bartenstein unter Berufung auf Montfaucon angeregt wurden ${ }^{73}$, später nach seinem Pariser Aufenthalt neuen Elan erhielten, aber auch etwa bei dem Maurinerschüler Marquard Herrgott ${ }^{74}$. In diesen Belangen ist ein gewisses Interesse bei Meichelbeck jedenfalls festzustellen, zumindest im Hinblick auf eine ordensinterne Studienreform. Dass er den erwähnten Brief Thibaults seinen Annalen inserierte, spricht dafür; seine eigene beigefügte Äußerung dazu, wie wünschenswert eine solche Studien-

71 LiEBOLD, Rokoko (wie Anm. 10) 25.

72 Vgl. Anm. 7; Hammermayer, Forschungszentren (wie Anm. 9) 153-165; Andreas KRAUS, Zur bayerischen Akademiebewegung im 18. Jahrhundert, in: Europäische Sozietätsbewegung und demokratische Tradition. Die europäischen Akademien der Frühen Neuzeit zwischen Frührenaissance und Spätaufklärung, hg. von Klaus Garber-Heinz WismanN-Winfried Siebers (Frühe Neuzeit 26/27, 2 Bde., Tübingen 1996) 2 1598-1616, hier 1600-1602; Alois SchMID, Das Augustiner-Chorherrenstift Polling und die Gründung der Bayerischen Akademie der Wissenschaften 1759 (Schriftenreihe der Akademie der Augustiner-Chorherren von Windesheim 10, Paring 2005).

73 Christine GlaSSNER, Bernard Pez et les tentatives pour créer une académie bénédictine en Autriche, in: Académies et sociétés savantes en Europe (1650-1800), hg. von Daniel-Odon Hurel-Gérard LAUdin (Colloques, congrès et conférences sur le Classicisme 1, Paris 2000) 491-507; vgl. WALLNIG-STOCKINGER, Korrespondenz 1 (wie Anm. 48) 9.

74 BAder, Sanct Blasien (wie Anm. 3) 181f.; Hammermayer, Forschungszentren (wie Anm. 9) 155f.; OrTner, Herrgott (wie Anm. 46) 44-46. 
ordnung für die bayerischen Benediktiner wäre, ließ es an Deutlichkeit nicht mangeln ${ }^{75}$. Schon einige Jahre früher hatte er ähnlich reges Interesse für einen Brief des Chorherren von Ste.-Geneviève Pierre-François Le Courayer gezeigt, den ihm der Pollinger Eusebius Amort ${ }^{76}$ mitteilte; darin ging es ebenfalls unter anderem um die in Paris gepflogene Studienordnung ${ }^{77}$. Dass Meichelbeck schon lange der scholastischen Lehrmethode abgeneigt war, wird im Folgenden gezeigt werden; er setzte auch später selbst Initiativen $^{78}$. An in den 1730er Jahren gegen einigen Widerstand in Gang gebrachten Bemühungen um eine Reform des Kommunstudiums der Bayerischen Kongregation war er in seinen letzten Lebensjahren noch beteiligt ${ }^{79}$. Wo es allerdings um Wissenschaftsorganisation über die Grenzen des Ordens hinweg ging, blieb Meichelbeck zurückhaltend: Als ihm 1722 die Mitarbeit an der Zeitschrift Parnassus Boicus angetragen wurde, lehnte er unter Angabe zu großer Arbeitsbelastung $\mathrm{ab}^{80}$.

Die Gründe dieses Abstandhaltens sind nicht eindeutig zu bestimmen. Die Entscheidung lag gewiss nicht bei Meichelbeck allein, sondern stets auch bei seinen Superioren. Unwillen zum fortgesetzten Kontakt mit dem

75 Abgedruckt bei BaumanN, Meichelbeck (wie Anm. 20) 49.

$76 \mathrm{Zu}$ ihm jetzt Karin Precht-NußBaum, Zwischen Augsburg und Rom. Der Pollinger Augustiner-Chorherr Eusebius Amort (1692-1775). Ein bedeutender Repräsentant katholischer Aufklärung in Bayern (Publikationen der Akademie der AugustinerChorherren von Windesheim 7, Paring 2007).

77 Der Brief Le Courayers abgedruckt bei VAN DülmEn, Anfänge (wie Anm. 55) 517-521; vgl. Precht-NubBaum, Zwischen Augsburg und Rom (wie Anm. 76) 150f. Zur Weitergabe an Meichelbeck vgl. BStB München, Meichelbeckiana 18b, 353v (22. Mai 1719); SiEGMUND, Meichelbecks Briefe (wie Anm. 43) 81 263-265 Nr. 36. Meichelbecks Eintrag dazu in den Kongregationsannalen zeigt freilich, dass sein größtes Interesse an dem Brief nicht Studienfragen, sondern Passagen zum Vorrangstreit zwischen Benediktinern und Regularkanonikern galt: HStA München, KL Benediktbeuern 125 I, 409r-410r.

78 Bei einem Besuch in Benediktbeuern während seiner Freisinger Zeit vermerkt Meichelbeck in seinem Diarium zum 11. Oktober 1726: Agimus de theologia aliter docenda (BStB München, Meichelbeckiana 18c, 188r); vgl. Stegmann, Desing (wie Anm. 8) 233f.

79 Wilhelm FinK, Beiträge zur Geschichte der bayerischen Benediktinerkongregation. Eine Jubiläumsschrift 1684-1934 (Studien und Mitteilungen zur Geschichte des Benediktiner-Ordens und seiner Zweige Ergh. 9, Metten-München 1934) 8890; HAering, Studien (wie Anm. 24) 127; ReichHold, 300 Jahre (wie Anm. 24) 566; Stegmann, Desing (wie Anm. 8) 234f., der die Einbeziehung Meichelbecks belegt.

80 Kolb, Autobiographie (wie Anm. 30) 56; vgl. Precht-NubBaum, Zwischen Augsburg und Rom (wie Anm. 76) 219f., die wohl mit Recht die Spannungen zwischen Benediktinern und Augustiner-Chorherren als Hintergrund vermutet; die wichtigsten Herausgeber des Parnassus waren Chorherren um Eusebius Amort. 
konfessionell oder theologisch Fremdartigen dürfte aber durchaus bei ihm selbst vorgelegen sein. Dies war wohl auch für seine Distanz zu den Maurinern mit ein Grund. 1716 hatte er im Briefwechsel mit Bernhard Pez noch darum gebeten, dass ihm dieser den Kontakt zu seinem Korrespondenten René Massuet vermitteln möge, dem er einige Fragen zur Geschichte der Karolinger unterbreiten wollte ${ }^{81}$. Zunächst war es der plötzliche Tod Massuets, der dieser Intention einen Riegel vorschob; Pez gelang jedoch rasch die Wiederaufnahme des Kontakts mit St.-Germain. 1717 allerdings schrieb Meichelbeck seinem Melker Freund, was er über die Haltung der Mauriner im Streit um die Konstitution Unigenitus erfahren habe, sei ihm unangenehm: „Gewiss war es bis jetzt in der Kirche Gottes und im Orden des heiligen Vaters Benedikt besser, dem Papst anzuhängen als den Königen. Haben etwa jene Herren Franzosen aus unserem Orden in ihren Codices etwas Gegenteiliges gefunden? ${ }^{\text {(82 } 2}$ Danach ist von einer Kontaktaufnahme nicht mehr die Rede.

\section{Entwicklung der Arbeitsweise Meichelbecks}

Was als Weg der Maurinerrezeption bei Meichelbeck bleibt, ist die Lektüre der Werke von Maurinergelehrten und deren Verwendung in und für seine eigenen Arbeiten ${ }^{83}$. Als letzter Teil der Untersuchung sollen daher im Folgenden die historischen Arbeiten Meichelbecks in einer - notgedrungen knappen - chronologischen Revue ins Auge gefasst werden. Seine erste tätige Berührung mit der Historie hatte er, soweit bekannt ist, während seiner Salzburger Studienzeit, als er von Paul Mezger ${ }^{84}$ als Amanuensis rekrutiert

81 Am 8. Januar 1716; abgedruckt bei SiEGMUND, Meichelbecks Briefe (wie Anm. 43) 80/1-2 150-152 Nr. 19; vgl. WeBER, Meichelbeck (wie Anm. 20) 131.

82 Stiftsarchiv Melk, Kt. 7 Patres 7, Bd. II, 690r-692v (Meichelbeck an Bernhard Pez, 9. Februar 1717): Sed quid hoc aliunde audio de Gallis nostris, quod scilicet notae pontificis constitutioni sese inter primos opponant? Certe hactenus in ecclesia Dei et in ordine sancti patris Benedicti praestabat adhaerere pontifici quam regibus. Repereruntne nostri illi domini Galli in suis codicibus quidquam contrarium? Abgedruckt bei SiEGMUND, Meichelbecks Briefe (wie Anm. 43) 80/1-2 170-173 Nr. 26; vgl. ebd. 81 270-272 Nr. 41, zur Haltung der Sorbonne gegenüber Unigenitus.

83 Dies betonen auch BAUMANN, Meichelbeck (wie Anm. 20) 32f.; BenZ, Zwischen Tradition und Kritik (wie Anm. 20) 602; MinderA, Jugend (wie Anm. 22) 103f;; vgl. Koch, Monachus eruditus (wie Anm. 20) 295f., 298; WüHR, Bedeutung (wie Anm. 27) 225-230.

84 Pirmin Lindner, Professbuch der Benediktiner-Abtei St. Peter in Salzburg (14191856). Mitteilungen der Gesellschaft für Salzburger Landeskunde 46 (1906) 1-328, 
wurde. Meichelbeck half dem Mitautor der Historia Salisburgensis ${ }^{85} 1694$ bei der Anfertigung von Streitschriften in der Kontroverse mit dem AugustinerChorherren Augustin Erath um die Passauer Exemption ${ }^{86}$.

Sehr bald nach seiner Rückkehr aus Salzburg wurde Meichelbeck die Leitung der Bibliothek anvertraut. Eine gerade einen Monat später datierte Diariumsnotiz vermerkt, dass er die Anschaffung von opera Mabillonii nostri vorschlug, welche allerdings Abt Eliland Öttl aus Kostengründen verweigerte $^{87}$. Um welche Werke es sich handelte, ist an dieser Stelle nicht zu ersehen. Die Schwierigkeit wurde nicht rasch ausgeräumt. Noch 1711 musste Meichelbeck das hilfswissenschaftliche Standardwerk De re diplomatica $^{88}$ aus Tegernsee entlehnen ${ }^{89}$, und erst 1716 konnte er nach langen Bemühungen Öttls Nachfolger Magnus Pachinger für den Ankauf von Mabillons Annales ordinis sancti Benedicti gewinnen ${ }^{90}$.

Nachweisbar ist bereits für die frühen Jahre auch, dass sich Meichelbeck intensiv der Durchsicht der Benediktbeurer Handschriften zuwandte.

hier 65-68; Rupert MitTERMÜLLER, Die Hauptvertreter der theologisch-philosophischen Wissenschaft an der Benedictiner-Universität Salzburg. Studien und Mittheilungen aus dem Benedictiner- und dem Cistercienser-Orden mit besonderer Berücksichtigung der Ordensgeschichte und Statistik 5/1 (1884) 122-148, hier 135-138; vgl. BENZ, Zwischen Tradition und Kritik (wie Anm. 20) 392; WALlnig, Modèle (wie Anm. 18) 301f.

85 [Joseph Mezger-Franz Mezger-Paul Mezger], Historia Salisburgensis, hoc est Vitae episcoporum et archiepiscoporum Salisburgensium nec non abbatum monasterii S. Petri ibidem (Salzburg 1692). Zur Salzburger Benediktinerhistoriographie vgl. den Beitrag von Barbara Lawatsch Melton in diesem Band.

86 MinderA, Jugend (wie Anm. 22) 79. Zum Streit: Thomas Stockinger, Felix mansurus, si tacuisset, Erath. Augustin Erath CRSA (1648-1719), Propst von St. Andrä an der Traisen, als Historiograph und historisch-politischer Kontroversist. Jahrbuch des Stiftes Klosterneuburg N. F. 20 (2008) 151-208, hier 161-172.

87 BStB München, Meichelbeckiana 18a, 146v (25. April 1696); MindERA, Jugend (wie Anm. 22) 96f., jedoch fälschlich zum 28. April 1696. Zu Öttl: Hemmerle, Benediktbeuern (wie Anm. 20) 525-529; LindNER, Professbuch Benediktbeuern (wie Anm. 20) 10f.

88 Jean MABILlon, De re diplomatica libri sex (Paris 1681).

89 BStB München, Meichelbeckiana 18b, 143v (14. Juni 1711); vgl. MindERA, Benediktbeurer Archiv (wie Anm. 25) 37.

90 BStB München, Meichelbeckiana 18b, 267v (9. Januar 1716): Vix non opus est strepitu, ut reverendissimus emat Annales nostri ordinis, qui iam ante multos annos in parvulis monasteriis fuerunt lecti; ebd. 283v (18. September 1716): Obtineo a reverendissimo tandem Annales nostri ordinis, quinque tomos. Es handelte sich also nicht bloß um den Nachkauf der zuletzt erschienenen Bände, sondern um das ganze Werk: Jean MABILLon et al., Annales ordinis sancti Benedicti (6 Bde., Paris 1703 1739), bis auf den erst viel später publizierten sechsten Band. $\mathrm{Zu}$ Pachinger: HEMMERLE, Benediktbeuern (wie Anm. 20) 529-535; LindnER, Professbuch Benediktbeuern (wie Anm. 20) 11-13. 
Im März 1697 gelang ihm in einem Codex der Bibliothek eine rechtlich bedeutsame Entdeckung, nämlich die Auffindung einer Notiz vom Anfang des 12. Jahrhunderts über eine Besitzteilung zwischen Benediktbeuern und Schlehdorf nahe dem Walchensee ${ }^{91}$. Da sein Kloster bereits seit Jahren mit dem benachbarten Augustiner-Chorherrenstift sowie mit den HieronymitanerEremiten, die auf dem strittigen Gebiet in den 1680er Jahren eine Niederlassung errichtet hatten, in einem für Benediktbeuern ungünstig verlaufenden Rechtsstreit lag ${ }^{92}$, war der Fund von keineswegs nur antiquarischem Interesse. Meichelbeck tadelte zwar seine Vorgänger, die von diesem Rechtstitel nichts geahnt hatten; der Fundort in einer wesentlich älteren Handschrift mit Gregors des Großen Regula pastoralis ${ }^{93}$ legt jedoch eher Zeugnis ab für seine eigene außerordentliche Genauigkeit.

Die Jahre am Freisinger Gymnasium dürften wenig Gelegenheit zu historischer Forschung gegeben haben, wohl aber vertrat Meichelbeck sein Kloster im Streit mit den Eremiten und in weiteren Rechtsgeschäften ${ }^{94}$. Die Berufung an das Kommunstudium, wo er erst Philosophie, dann Theologie $\mathrm{zu}$ unterrichten hatte, war insoweit auch nicht gerade eine Einladung zur Geschichtsforschung. Meichelbeck brachte diese allerdings ein, wo sie gar nicht vorgesehen war. Die monatlichen Exercitationes, die er seinen Studenten zu disputieren gab - zugleich seine ersten gedruckten Arbeiten - sind schon frühzeitig als Vorzeichen seiner späteren Leistungen gewertet worden. Sein Schüler und Biograph Alphons Haidenfeld etwa schrieb darüber: „Die philosophischen und theologisch-spekulativen Feinheiten vermischte er nämlich in solcher Weise mit soliden dogmatischen, historischen, kritischen und dergleichen Fragen, dass sie auch denen nützlich und angenehm sein würden, denen nicht so sehr die scholastischen Widerwärtigkeiten als viel-

91 BStB München, Meichelbeckiana 18a, 174v (28. März 1697): Invenis in bibliotheca vetustissimum manuscriptum de divisione nemoris in peninsula Wallerseensi facta circa annum Domini 1100. Stupes cum aliis, quod ii, quos concerneret res ista, similia penitus non investigent; vgl. MindERA, Jugend (wie Anm. 22) 97.

92 Hermann HöRGER, Geistliche Grundherrschaft und nachtridentinisches Frömmigkeitsbedürfnis. Die Abtei Benediktbeuern im Kampf gegen das Eremitorium Walchensee (1687-1725). Zeitschrift für bayerische Kirchengeschichte 47 (1978) 69-84; Anton ScHMID, Die Nachblüte der Abtei Benediktbeuern nach dem dreißigjährigen Kriege. Studien und Mitteilungen zur Geschichte des Benediktiner-Ordens und seiner Zweige 42 (1924) 71-156, hier 94f.; vgl. HemmerLe, Benediktbeuern (wie Anm. 20) 236f., 532; WeBER, Meichelbeck (wie Anm. 20) 135f.; WÜHR, Bedeutung (wie Anm. 27) 229f.

93 BStB München, clm 4614, 143r-v; vgl. Günter GlaUCHE, Catalogus codicum manu scriptorum Bibliothecae Monacensis, 3/Series Nova 1: Katalog der lateinischen Handschriften der Bayerischen Staatsbibliothek München. Die Pergamenthandschriften aus Benediktbeuern Clm 4501-4663 (Wiesbaden 1994) 200.

94 MinderA, Jugend (wie Anm. 22) 98. 
mehr wahrhaftigere Gelehrsamkeit und Kenntnis kirchlicher und monastischer Geschichte am Herzen lagen“"95. Auch neuere Autoren haben diese Arbeiten als bedeutsam eingestuft ${ }^{96}$, ja sogar als „entscheidenden Wendepunkt zum Geschichtsfach bei dem herangereiften Gelehrten“ gedeutet ${ }^{97}$. Ein näherer Blick in diese sich über einen Zeitraum von etwa fünf Jahren erstreckenden Schriften dürfte sich also lohnen.

Ab der dritten philosophischen Übung von 1702 wird die von Haidenfeld angesprochene Mischung von scholastischen Thesen und historischen Inhalten erkennbar. Diese Exercitatio handelt von Wesen und Eigenschaften der Logik; die einzelnen Punkte bestehen jedoch in der Regel aus einem einzigen Satz, der in den Begrifflichkeiten der scholastischen Philosophie eine dieser Eigenschaften beschreibt, gefolgt von langen historischen Ausführungen über das Vorliegen derselben Eigenschaft beim Hl. Benedikt und seinem Orden. Etwa beginnt die elfte Position: „Wir halten die wissenschaftliche Logik für den anderen Wissenschaften überaus nützlich, ja sogar für schlichtweg notwendig, um diese im vollendeten Stande zu erlangen. $\mathrm{Du}$ fragst, ob der Orden unseres heiligen Vaters Benedikt für die Kirche Gottes nützlich gewesen ist? Ich bejahe es“. Darauf folgen vier Seiten Darlegungen über benediktinische Verdienste um die Kirche, die mit der Logik nichts zu tun haben ${ }^{98}$.

Ähnlich sind auch die folgenden Exercitationes aufgebaut. Die historischen Ausführungen sind an die philosophischen Positionen zumeist nur über ein einzelnes Wort angeknüpft, wobei dessen präzise definierte scholastische Bedeutung nicht respektiert, ja teils geradezu abgetan wird. In der fünften Exercitatio handelt die zweite Position von den unterschiedlichen Arten der Einheit: unitas generica, specifica, numerica, praecisionis; hervorragender als alle diese, heißt es dann aber sofort mit dem Epheserbrief, sei die „Einheit des Geistes durch das Band des Friedens ${ }^{\text {‘99 }}$. Und da diese

95 Alphons HAIDENFeld, Vita autoris reverendi patris Caroli Meichelbeckii monachi Benedicto-Burani, in: MeICHelBeck, Chronicon Benedictoburanum (wie Anm. 29) L-LXVIII, hier LII: Philosophicas enim et theologico-speculativas subtilitates quaestionibus solidis dogmaticis, historicis, criticis etc. sic temperavit, ut ad usum et voluptatem etiam essent iis, quibus non tam scholarum tricae quam sincerior eruditio et historiae tum ecclesiasticae, tum monasticae notitia cordi et curae essent.

96 Baumann, Meichelbeck (wie Anm. 20) 12; WüHR, Bedeutung (wie Anm. 27) 229.

97 MindERA, Jugend (wie Anm. 22) 99.

98 Karl MeichelBeck, Exercitatio tertia menstrua sive Positiones de natura et proprietatibus logicae (Augsburg 1702) (unpag.): XI. Logicam artificialem aliis scientiis perquam utilem, imo ad eas in statu perfecto aquirendas simpliciter necessariam existimamus. - Quaeris, fueritne ordo sancti patris nostri Benedicti ecclesiae Dei utilis? Affirmo.

99 Karl MeICHELBeCK, Exercitatio quinta menstrua sive Positiones de unitate, identitate et distinctione (München 1702) 2: II. Unitas alia est generica, alia specifica, alia 
Exercitatio den Schutzengeln gewidmet ist, unter deren Patrozinium die Bayerische Kongregation stand, folgen Berichte über den freundschaftlichen Verkehr von Engeln mit Benediktinerheiligen. Wenn schließlich in der sechsten Exercitatio, zugeeignet den Hl. Petrus und Paulus, eine Position lautet, ein universale metaphysicum sei definiert als unum aptum inesse pluribus, und es dann heißt, die Leiber der Apostelfürsten könnte man beinahe als universalia metaphysica bezeichnen, weil sie nicht an einem einzigen Ort seien, sondern auf so viele - besonders benediktinische - Kirchen verteilt, dann ist wohl die Grenze vom Desinteresse an der Schulphilosophie zur Parodie derselben überschritten ${ }^{100}$. Unklar bleibt freilich, was die Studenten zu verteidigen hatten: die Positionen allein oder auch Meichelbecks historische Assoziationen dazu. Die erhaltenen Vorlesungsmitschriften weisen allerdings auf Ersteres, denn sie zeigen, dass Meichelbeck eine mehr oder minder übliche Logikvorlesung ohne ordensgeschichtliche Exkurse vorgetragen hatte ${ }^{101}$.

Die Art der Beweisführung in den historischen Ausführungen ist im Übrigen nicht unbedingt quellenkritisch. Für das Benediktinertum des Hl. Rupert wird als Beleg angeführt, dass ihn der Salzburger Erzbischof Paris Lodron im Dom im Ordenshabit habe malen lassen ${ }^{102}$; für eine Begebenheit im Leben des Hl. Benedikt wird eine Bulle Urbans VIII. allegiert ${ }^{103}$. Zitiert werden Johannes Trithemius ${ }^{104}$ und immer wieder Gabriel Bucelin zu den verschiedensten Themen ${ }^{105}$, aber keine handschriftlichen Quellen. Viele Tat-

numerica, alia praecisionis, quarum notiones ita abstrusae non sunt, ut dilucidari hoc loco debeant. - His tamen omnibus praecellentior est unitas spiritus, quae fundatur in vinculo pacis, ut habet apostolus; vgl. Eph 4,3.

100 Karl MeichelBeCK, Exercitatio sexta menstrua sive Positiones de universalibus in genere (München 1702) 2: II. Universale ad nostrum propositum definiri rigidius solet, quod sit unum aptum inesse pluribus; et haec est definitio universalis metaphysici. [...] Caeterum sacratissima divorum apostolorum Petri et Pauli corpora pene dixeris quaedam universalia metaphysica, eo quod sint in loco non uno.

101 BStB München, clm 4853 und 4854; vgl. DACHS, Meichelbeckiana (wie Anm. 39) 201-203.

102 MeICHelBeck, Exercitatio tertia (wie Anm. 98) (unpag.): Illa ipsa Salisburgensium metropolitana sedes cui alii adscribenda est quam sanctissimo Ruperto nostro, primo S. Petri ibidem abbati, quem idcirco Benedictino habitu pingi in moderna metropolitana ecclesia voluit sapientissimus ille Paris Lodronius archiepiscopus rei veritate convictus.

103 MeichelBeCK, Exercitatio tertia (wie Anm. 98) (unpag.).

104 Meist ohne Angabe konkreter Titel, aber wohl vor allem aus De viris illustribus ordinis sancti Benedicti schöpfend. Zu Trithemius sei nur verwiesen auf Klaus ARNOLD, Johannes Trithemius (1462-1516) (Quellen und Forschungen zur Geschichte des Bistums und Hochstifts Würzburg 23, Würzburg $\left.{ }^{2} 1991\right)$.

$105 \mathrm{Zu}$ ihm: Claudia Maria NeESEN, Gabriel Bucelin OSB (1599-1681). Leben und historiographisches Werk (Stuttgarter historische Studien zur Landes- und Wirt- 
sachenbehauptungen stehen ganz ohne Beleg da, darunter reichlich fragwürdige, etwa dass Alkuin 790 die Pariser Universität gegründet habe ${ }^{106}$. Der erwähnte Absatz über den Verkehr der Benediktinerheiligen mit den Engeln - dem Hl. Dunchad reichten diese Brot und Wein, Rainald von Nocera legten sie beim Gottesdienst Ärmel an, mit dem Hl. Gudwal sangen sie das Stundengebet, Abt Wandregisel wischten sie vor den Augen des Königs Dagobert I. den Schmutz vom Gewand und so fort - erweist sich bei Nachschau als zur Gänze aus dem Menologium Benedictinum von Bucelin zusammengestellt ${ }^{107}$, das Meichelbeck jedoch nicht zitiert. Der Philosophieprofessor von 1702 zeigt zwar ein unverhohlenes Missfallen an der scholastischen Lehre und den Wunsch, von ihr zugunsten einer historischpositiven Argumentation abzugehen; von „maurinischer Methode“ lässt er allerdings wenig erkennen.

Einige Jahre später, in den theologischen Disputationen von 1706 und 1707, verhält sich dies bereits deutlich anders. Der Aufbau ist weiterhin ähnlich, wenn er auch expliziter präsentiert wird, indem unter getrennten Überschriften Assertiones theologicae und Allusiones historicae abwechseln. Meichelbeck widmet eine jede dieser Exercitationes einem oder einer regionalen Heiligen - Ulrich, Afra, Korbinian, Anastasia und so weiter -, dessen oder deren Leben er untersucht, und zwar nun bereits unter Heranziehung und kritischem Vergleich handschriftlicher Überlieferungen. In der dritten Exercitatio über Korbinian etwa diskutiert er die Frage nach dessen Herkunft. Für die widerstreitenden Meinungen, die Heimat des Heiligen liege in Châtres ${ }^{108}$ bei Melun in Frankreich oder bei Mais unweit Meran in

schaftsgeschichte 3, Ostfildern 2003); vgl. BENZ, Zwischen Tradition und Kritik (wie Anm. 20) 146f., 557-560, 618f.

106 MeichelBeCK, Exercitatio tertia (wie Anm. 98) (unpag.).

107 Gabriel Bucelin, Menologium Benedictinum sanctorum, beatorum atque illustrium eiusdem ordinis virorum elogiis illustratum (Feldkirch 1655), insb. 47 (Dunchad), 108f. (Rainald), 405 (Gudwal), 512f. (Wandregisel). - Die Vorstellung, ein quellenkritischer Blick auf die historische Vergangenheit sei notwendigerweise mit Wunderglauben unvereinbar, ist eine Rückprojektion von Historiographiehistorikern des 19. und 20. Jh., die sich mit Blick auf die Produktion frühneuzeitlicher Gelehrter nicht halten lässt; vgl. zu den Bollandisten Jan Marco SAwILlA, Antiquarianismus, Hagiographie und Historie im 17. Jahrhundert. Zum Werk der Bollandisten. Ein wissenschaftshistorischer Versuch (Frühe Neuzeit. Studien und Dokumente zur deutschen Literatur und Kultur im europäischen Kontext 131, Tübingen 2009) 44-53, 427f., 523-535; zu Mabillon: Daniel-Odon Hurel, Jean Mabillon, Jean-Baptiste Thiers et la congrégation de Saint-Maur, in: Dom Jean Mabillon (wie Anm. 16) $59-76$.

108 Heute Arpajon im Département Essonne; nicht zu verwechseln mit Chartres im Département Eure-et-Loir. 
Südtirol, nennt er die je ältesten Belege - Arbeos Vita Corbiniani ${ }^{109}$ respektive ein bei Wiguleus Hund zitiertes Freisinger Traditionsbuch ${ }^{110}$ - und zeichnet die Abhängigkeit aller späteren Autoren von diesen Quellen nach, bevor er sich für die Tiroler Herkunft entscheidet ${ }^{111}$. In der Frage der Herkunft der Stifter Benediktbeuerns, die unmittelbar daran anschließt, führt er mehrere verschiedene Codices der Klosterbibliothek als Quellen unterschiedlicher Versionen an, wiederum unter Angabe der neueren Autoren, die ihnen gefolgt $\operatorname{sind}^{112}$. Die Entscheidung zwischen diesen divergieren Überlieferungen erfolgt aufgrund ihrer inhaltlichen Plausibilität, insbesondere der chronologischen Widersprüche, die Meichelbeck den Erzählungen nachweist; die von ihm für richtig erachtete Version, wonach Landfrid, Waldram und Eliland dem agilolfingischen Herrscherhaus entstammten, ist freilich gerade diejenige, die er am wenigsten genau mit handschriftlichen Quellen belegt. Vielmehr beruft er sich auf Aventin, Hund, Aubert Le Mire und Buce$\operatorname{lin}^{113}$ und setzt dann nur noch die knappe Aussage hinzu, diese Meinung stimme mit den ältesten Benediktbeurer Dokumenten überein, „,soweit diese nicht durch die so zahlreichen und schweren Unglücksfälle im Verlauf der Zeit verfälscht worden sind“"114. Eine hilfswissenschaftliche Untersuchung der Quellen leistet Meichelbeck nicht, ja er erwähnt nichts in dieser Richtung,

109 In der Edition von Jean-Luc d'ACHERY-Jean MABILLON et al., Acta sanctorum ordinis sancti Benedicti in saeculorum classes distributa (9 Bde., Paris 1668-1701) 3/1 500-517, hier 501; vgl. Lothar Vogel, Vom Werden eines Heiligen. Eine Untersuchung der Vita Corbiniani des Bischofs Arbeo von Freising (Arbeiten zur Kirchengeschichte 77, Berlin-New York 2000) 42f.

110 Wiguleus Hund vON SulzenMOOS-Christoph GEwOld, Metropolis Salisburgensis (3 Bde., München 1620) 1 101. Die zitierte Quelle ist das Traditionsbuch des Sakristans Konrad von 1187, heute HStA München, HL Freising 3c; vgl. Theodor BITTERAUF, Die Traditionen des Hochstifts Freising, 1: (744-926) (Quellen und Erörterungen zur bayerischen und deutschen Geschichte N. F. 4, München 1905) XXIIXXV; Markus MüLLER, Die spätmittelalterliche Bistumsgeschichtsschreibung. Überlieferung und Entwicklung (Beihefte zum Archiv für Kulturgeschichte 44, Köln-Weimar-Wien 1998) 224-226.

111 Karl MeichelBECK, Exercitatio tertia menstrua theologico-historica sive Positiones ex Tractatu de iure et iustitia, quas magni Frisingensium patris et pontificis Corbiniani virtutibus et meritis interpolatas [...] (Regensburg 1707) 7-9.

112 MeICHELBECK, Exercitatio tertia theologico-historica (wie Anm. 111) 9-14.

113 Johann Turmair [Aventinus], Annalium Boiorum libri septem (Ingolstadt 1554) 292; Hund VON SulzenMoos-Gewold, Metropolis Salisburgensis (wie Anm. 110) 2 139; Aubert LE MiRE, Origines Benedictinae (Köln 1614) 175; Gabriel BuCELIN, Germania topo-chrono-stemmatographica sacra et profana (Augsburg 1655) 278.

114 Meichelbeck, Exercitatio tertia theologico-historica (wie Anm. 111) 14: Nostris sane antiquissimis documentis (ex qua parte per tot tamque acerbas temporum vicissitudines vitium non acceperunt) accuratissime haec sententia consonat. 
obwohl sie ihm im zweiten Falle durchaus verfügbar waren ${ }^{115}$. Unter den zitierten Schriftstellern ist übrigens auch Mabillon mit seinen Vetera analecta ${ }^{116}$, freilich unter denen, deren Meinung abgelehnt wird.

In den folgenden Jahren hatte Meichelbeck wenig Gelegenheit, sich mit ähnlichen Fragen zu beschäftigen, dafür viel mit der Zeitgeschichte zu tun. 1708 übertrug ihm das Generalkapitel die Führung der Kongregationsannalen; er zeigte hierbei mehr Initiative und Konsequenz als irgendeiner seiner Vorgänger und Nachfolger. Die fortlaufende Aufzeichnung der Geschäfte der Kongregation und ihrer Klöster erfolgte aufgrund von diesen eingesandter Berichte; deren Sammlung systematisierte und betrieb Meichelbeck mit Nachdruck ${ }^{117}$. In seinem Kloster wurde ihm die Aufsicht über das Archiv übertragen, dessen vollständige Neuordnung er in Angriff nahm. Große Teile der Bestände wurden bis auf die Ebene von Einzelstücken erschlossen und einsigniert. Das dabei erstellte Inventar füllt vier Bände mit raffinierten Indizes ${ }^{118}$. Diese Arbeiten flossen in die Rechtsangelegenheiten des Klosters ein; im Streit mit den Eremiten am Walchensee wurde Meichelbeck 1713 nach Rom entsandt und konnte dort den Gerichtsfall zugunsten Benediktbeuerns entscheiden ${ }^{119}$. Danach legte er den Hergang seit der Ansiedlung der Hieronymitaner 1687 in einer handschriftlichen Histori deß eremitory am Wallersee nieder ${ }^{120}$. Auch der Streit mit Schlehdorf wurde 1716 durch einen Vergleich beigelegt, an dem Meichelbeck entscheidend mitwirkte ${ }^{121}$; überhaupt wurde er vielfältig in Rechtsgeschäften seines Klosters und der Kongregation eingesetzt. Für den Fürstabt von Kempten Rupert von Bodman, mit dem seit der Zeit am Freisinger Gymnasium Kontakt bestand ${ }^{122}$, verfasste Meichelbeck pseudonyme Streitschriften im Streit mit dessen Kapitel

115 Das Kommunstudium befand sich 1707 in Benediktbeuern: REICHHOLD, 300 Jahre (wie Anm. 24) 672.

116 Jean MABILlON, Veterum analectorum tomi quatuor (Paris 1675-1685) 4 533f.

117 Siegmund, Annales (wie Anm. 26) 153-158; vgl. SiEgmund, Meichelbecks Briefe (wie Anm. 43) 80/1-2 137f. Nr. 14, 146-150 Nr. 18; 81 269f. Nr. 40, 281-288 Nr. 46-52, 296-298 Nr. 60-61; WALLNIG-STOCKINGER, Korrespondenz 1 (wie Anm. 48) 775-780 Nr. 474.

118 DACHS, Meichelbeckiana (wie Anm. 39) 197f.; MinderA, Benediktbeurer Archiv (wie Anm. 25) 43-46. Zu den Verflechtungen zwischen Archivpraxis und Historiographie vgl. den Beitrag von Helga Penz in diesem Band.

119 Vgl. Anm. 92; sowie Wallnig-Stockinger, Korrespondenz 1 (wie Anm. 48) 755-761 Nr. 464

120 BStB München, Meichelbeckiana 16; vgl. DACHS, Meichelbeckiana (wie Anm. 39) $198 f$.

121 BStB München, Meichelbeckiana 18b, 275v, 277v, 286r-289r; vgl. SIEGMUND, Meichelbecks Briefe (wie Anm. 43) 80/1-2 170-173 Nr. 26.

122 MinderA, Jugend (wie Anm. 22) 98; zu Bodman: Joseph Rottenkolber, Geschichte des hochfürstlichen Stiftes Kempten (München 1933) 152-166. 
um die Wahl eines Koadjutors; auch hier bestand seine Aufgabe nach eigener Schilderung darin, große Aktenberge in kürzester Zeit zu sichten und zu einer Darstellung zusammenzufassen ${ }^{123}$.

Das bereits mehrfach ${ }^{124}$ unterbreitete Angebot seines langjährigen Förderers, des Bischofs Eckher, zur Verfassung einer Freisinger Diözesangeschichte wurde 1722 schließlich in einer Weise erneuert, die keine Ablehnung mehr gestattete $^{125}$. Damit begann für Meichelbeck ein fünfjähriger Aufenthalt am Freisinger Hof, der nicht immer angenehm verlief, denn der Gunst des Bischofs stand eine misstrauische bis ablehnende Haltung des Domkapitels gegenüber, das aus der Publikation der Urkunden Nachteile fürchtete und Meichelbecks Darstellung des Gründers Korbinian und der frühen Klerikergemeinschaft der Domkirche als Benediktiner beeinspruchte ${ }^{126}$. Auch die Inanspruchnahme durch die Bayerische Benediktinerkongregation reduzierte sich kaum ${ }^{127}$.

Meichelbecks Verbindungen im Rahmen der Kongregation und seine Erfahrungen bei der Einholung der Berichte für die Annalen dürften allerdings hilfreich gewesen sein für eines der hervorstechenden Merkmale der Historia Frisingensis, nämlich die weit ausgreifende Quellensammlung. Neben Freisinger Beständen und den ihm gut bekannten Benediktbeurer

123 Karl Meichelbeck [pseud. Georgius a Sancto Carolo], Relatio super celebri controversia inter quosdam illustrissimos et reverendissimos dominos capitulares aliosque de reverendissimo conventu Campidonensi Romae vertente in puncto praetensae vocis capitularis (Kempten 1719); Karl MEICHELBECK [pseud. GEORGIUS A SANCTO CAROLO], Relatio altera super celebri controversia inter quosdam illustrissimos et reverendissimos dominos capitulares aliosque de reverendissimo conventu Campidonensi in puncto praetensae vocis capitularis (Kempten 1720); vgl. SIEGMUND, Meichelbecks Briefe (wie Anm. 43) 81 261f. Nr. 34, 270-272 Nr. 41, 273276 Nr. 43. Zum Streit vgl. Joseph RotTEnKOlBer, Der Koadjutorstreit unter dem Kemptner Fürstabt Rupert von Bodmann. Zeitschrift für bayrische Kirchengeschichte 2 (1927) 34-41, 154-161.

124 Erstmals bereits 1709: Baumann, Meichelbeck (wie Anm. 20) 13f.; Mindera, Jugend (wie Anm. 22) 102; WüHR, Bedeutung (wie Anm. 27) 233.

125 BStB München, Meichelbeckiana 18c, 59r (7. März 1722): Literas prorsus singulares a reverendissimo cum inclusis celsissimi Frisingensis. [...] Evocor Frisingam. Deus esto mihi propitius!

126 Vgl. Baumann, Meichelbeck (wie Anm. 20) 16f.; Hubensteiner, Geistliche Stadt (wie Anm. 13) 184f., 188f.; WÜHR, Bedeutung (wie Anm. 27) 234, $236 f$.

127 Präses der Kongregation war während dieser Jahre Abt Ildephons Huber von Weihenstephan. Das Diarium weist eine dichte Folge von Besuchen Meichelbecks in dessen unmittelbar bei Freising gelegenem Kloster aus. Zu Huber vgl. Heinrich GENTNER, Geschichte des Benedictinerklosters Weihenstephan bey Freysing. Aus Urkunden angefertiget. Beyträge zur Geschichte, Topographie und Statistik des Erzbisthums München und Freysing 6 (1854) 1-350, hier 157-166, 313-324. 
Quellen konnte Meichelbeck noch aus einer beträchtlichen Zahl von Klöstern $^{128}$ der Region die Übermittlung von Abschriften, oft aber auch von Codices im Original erwirken - genannt sei etwa das Verbrüderungsbuch von St. Peter in Salzburg ${ }^{129}$. In der Analyse dieser Quellen war er über das Niveau seiner letzten einschlägigen Veröffentlichung in den theologischen Exerzitationen deutlich hinausgelangt, wie sich an einigen Beispielen zeigen lässt.

Etwa rollte Meichelbeck hier die Frage der Herkunft Korbinians wieder auf - und revidierte seine frühere Ansicht ${ }^{130}$. Es ging immer noch um die Entscheidung zwischen denselben zwei Quellen, doch kannte er diese inzwischen genauer - nämlich beide aus eigener Anschauung und nicht mehr aus Editionen oder Zitaten - und war vor allem neuer Bewertungskriterien gewahr worden. Zum einen pochte er, was er in den Exercitationes keineswegs durchgehend getan hatte, nun auf zeitliche Nähe als wichtigsten Grund der Glaubwürdigkeit: „In diesem Streitpunkt halten wir uns mit Recht an Arbeo, den wohlgemerkt ersten Biographen des Hl. Korbinian, von dem, so wie er näher als alle anderen Schriftsteller an dem Hl. Korbinian lebte und dessen Heimat und übrige Taten vollständiger kennen konnte, ebenso auch durchaus angenommen werden muss, dass er getreulich berichtete “131. Zum anderen standen ihm inzwischen zur Altersbestimmung von Quellen zusätzliche, nämlich hilfswissenschaftliche Methoden zu Gebote; in der Historia Frisingensis datiert er geläufig nach paläographischen Kriterien in der von Mabillon propagierten Weise, also etwa manu saeculi XIII. exarata und dergleichen ${ }^{132}$. Gerade die Freisinger Traditionsbücher hatte er eingehend

128 Meichelbeck selbst nennt, abgesehen vom Domkapitel, 17 Institutionen, die Quellen bereitgestellt hatten (Benediktiner, Augustiner-Chorherren, Prämonstratenser, Jesuiten, Säkularkanonikerstifte): MEICHELBECK, Historia Frisingensis (wie Anm. 28) $1 / 1$ XXXVIII f.

129 Vgl. Karl ForstneR, Das Verbrüderungsbuch von St. Peter in Salzburg. Vollständige Faksimile-Ausgabe im Originalformat der Handschrift A 1 aus dem Archiv von St. Peter in Salzburg (Codices selecti phototypice impressi 51, Graz 1974) 15f.

130 Meichelbeck, Historia Frisingensis (wie Anm. 28) 1/1 XVI-XX; vgl. Vogel, Vom Werden eines Heiligen (wie Anm. 109) $75 \mathrm{f}$.

131 MeICHELBECK, Historia Frisingensis (wie Anm. 28) 1/1 XVIII: In hoc dissidio merito ad Aribonem recurrimus, primum scilicet divi Corbiniani biographum, [...] qui, uti caeteris scriptoribus omnibus propinquior sancto Corbiniano vixit ac patriam et reliqua illius acta plenius scire potuit, ita etiam fideliter retulisse omnino censendus est.

132 Hier MeIchelbeck, Historia Frisingensis (wie Anm. 28) 1/1 XXXV. Die Datierung nach Jahrhunderten war durch die Werke Mabillons und Montfaucons seit etwa 1675 propagiert worden: Paul LeHMANN, Einteilung und Datierung nach Jahrhunderten, in: Aus der Geisteswelt des Mittelalters. Studien und Texte Martin GraBMANN zur Vollendung des 60. Lebensjahres von Freunden und Schülern gewidmet, hg. von 
untersucht und wusste daher, dass dasjenige, in dem die Tiroler Herkunft behauptet wurde, um Jahrhunderte jünger ist als Arbeos Vita. Die Passage seiner Vorbemerkungen, in der er sechs verwendete Traditionsbücher der Reihe nach vorstellt, ist bemerkenswert: Er liefert Datierungen, Bemerkungen zur Scheidung von Schreiberhänden, Angaben zu Art und Zahl der Notizen sowie Beobachtungen zur Entstehungsgeschichte und zeigt damit nicht nur seine Fähigkeit, den Codices dies zu entnehmen, sondern auch die nicht selbstverständliche Annahme, dass diese Informationen für die Leser von Interesse sein werden ${ }^{133}$.

An Schriftstellern zitiert Meichelbeck nunmehr bevorzugt neuere und neueste Literatur, so die Editionen von Bernhard und Hieronymus $\mathrm{Pez}^{134}$, die Critica von Antoine Pagi ${ }^{135}$, Korbinian Khamms Hierarchia Augustana ${ }^{136}$, die Historia Salisburgensis der Brüder Mezger ${ }^{137}$, die Historiographen Bayerns Johann Adlzreiter und Andreas Brunner ${ }^{138}$, und natürlich Mabillon: vielfach die Annales und Acta sanctorum ordinis sancti Benedicti, gelegentlich die Vetera analecta ${ }^{139}$, punktuell auch De re diplomatica und dessen

Albert LANG-Josef LeChNER-Michael Schmaus (Beiträge zur Geschichte der Philosophie und Theologie des Mittelalters. Texte und Untersuchungen Supplementband 3, 2 Bde., Münster 1935) 1 35-51, hier 50f. Gerade für das frühe 18. Jh. kann sie daher als Indiz für Maurinerrezeption gewertet werden; vgl. François DoLbEAU, Les sources manuscrites des Acta Sanctorum et leur collecte (XVII ${ }^{\mathrm{e}}-\mathrm{XVIII}$ siècles), in: De Rosweyde aux Acta Sanctorum. La recherche hagiographique des Bollandistes à travers quatre siècles. Actes du Colloque international (Bruxelles, 5 octobre 2007), hg. von Robert GodDING-Bernard JoASSART-Xavier LEQUEUX-François DE VRIENDT (Subsidia hagiographica 88, Bruxelles 2009) 105-147, hier 108f.

133 MeICHELBeCK, Historia Frisingensis (wie Anm. 28) 1/1 XXXIV-XXXVI.

134 Bernhard PEZ, Thesaurus anecdotorum novissimus (6 Bde., Augsburg-Graz 17211729); Hieronymus PEZ, Scriptores rerum Austriacarum veteres ac genuini (3 Bde., Leipzig-Regensburg 1721-1745); vgl. Christine GlassNer, Der ,Thesaurus anecdotorum novissimus" des Melker Benediktiners Bernhard Pez. Studien und Mitteilungen zur Geschichte des Benediktinerordens und seiner Zweige 113 (2002) 341370.

135 Antoine PAgI, Critica historico-chronologica in Annales ecclesiasticos Caesaris Baronii (Paris 1689).

136 Korbinian Кнамм, Hierarchia Augustana tripartita in partem cathedralem, collegialem et regularem (5 Bde., Augsburg 1709-1719). Khamm, Benediktiner von St. Ulrich und Afra zu Augsburg, war ein langjähriger Korrespondent Meichelbecks; vgl. MinderA, Jugend (wie Anm. 22) 100.

137 Mezger-MezGer-Mezger, Historia Salisburgensis (wie Anm. 85).

138 Johann AdLzREITER [recte: Johann VerVAuX], Boicae gentis annalium partes tres (München 1662-1663); Andreas BRUNNER, Annales virtutis et fortunae Boiorum (3 Bde., München 1626-1637).

139 Mabillon et al., Annales ordinis sancti Benedicti (wie Anm. 90); ACHERYMABILLON et al., Acta sanctorum (wie Anm. 109); MABILLON, Vetera analecta (wie 
Supplement, jedoch für einzelne Fakten, nicht für methodische Aussagen ${ }^{140}$. Grundsätzlich vertraut er allerdings keinem Autor und stützt sich auf diese nur, wo keine handschriftlichen Quellen zur Verfügung stehen: „Im Übrigen wird, wo wir unsere Angaben nicht auf Dokumente und Urkunden, sondern nur auf die Vertrauenswürdigkeit und Autorität des einen oder anderen Schriftstellers stützen, der kluge Leser leicht begreifen, dass wir für derartige Zeugnisse nicht mehr Glaubwürdigkeit behaupten, als die Gelehrten ihnen beizulegen pflegen. Was uns freilich zweifelhaft erscheint, haben wir in solchen Formulierungen ausgedrückt, aus denen niemand nicht ersehen müsste, dass wir zu diesen Ereignissen nichts Bestimmtes entscheiden wollen ${ }^{\text {“141 }}$. Diese Priorität der Primärquellen bestimmt auch die Darstellung, in der für manche Perioden seitenlang kein Literaturzitat erscheint, vielmehr jedoch die Margen dicht besetzt sind mit Nummernverweisen auf den Quellenband. Dieser, die Pars instrumentaria, umfasst nicht weniger als 1385 Nummern allein für die ersten 500 Jahre Freisings. Dieses Vorgehen schien auch Meichelbeck selbst, der mit expliziten Aussagen zur Methodik sonst nicht großzügig war, gegenüber dem Publikum einer Begründung zu bedürfen. In einer eigenen Vorrede rechtfertigte er nicht nur den Umfang der Quellensammlung, sondern auch in einer genuin antiquarischen Argumentation seine Entscheidung, die Latinität der Vorlagen zu respektieren: „Wir schreiben hier nicht, um Grammatik zu unterrichten oder nach sprachlicher Anmut zu streben, sondern um das reine und wahrhafte Altertum und die Kenntnis des Geschehenen ans Licht zu bringen“142.

Mit der Historia Frisingensis war Meichelbeck somit - in diesem Punkt können die Wahrnehmungen der Zeitgenossen wie die Urteile der bisherigen Historiographiegeschichte nur bestätigt werden - dem fortgeschrittensten zeitgenössischen Stand in der Entwicklung der historisch-kritischen

Anm. 116). Zitate aus dem letzteren Werk etwa bei MEICHELBECK, Historia Frisingensis (wie Anm. 28) 1/1 XXII f., 47, 136; 1/2 23.

140 Mabillon, De re diplomatica (wie Anm. 88); Jean Mabillon, Librorum de re diplomatica supplementum (Paris 1704). Ersteres zitiert etwa bei MEICHELBECK, Historia Frisingensis (wie Anm. 28) 1/1 174; Letzteres ebd. 1/2, Praefatio (unpag.).

141 MeIChelbeck, Historia Frisingensis (wie Anm. 28) 1/1 XXXIX f.: Caeterum ubi assertiones nostras non documentis et instrumentis, sed sola unius vel alterius scriptoris fide et autoritate communimus, facile intelliget prudens lector nos eiusmodi testimoniis non postulare maiorem fidem ac eam, quam solent adhibere eruditi. Ea sane, quae nobis visa fuere dubia, iis scribendi modis expressimus, ex quibus nemo non videre debeat nos circa eiusmodi eventus nihil certi statuere velle.

142 Meichelbeck, Historia Frisingensis (wie Anm. 28) 1/2, Praefatio (unpag.): Scribimus hic non grammaticam docendo et sermonis veneres consectando, sed puram ac germanam antiquitatem ac rerum gestarum notitiam eruendo. Vgl. zu dieser Argumentation SAwILlA, Antiquarianismus (wie Anm. 107) 395-425. 
Methode sehr nahe gekommen. Die Werke Mabillons waren ihm dabei sichtlich wichtige Hilfsmittel. Bevor er aber deshalb zum „deutschen Mauriner" erklärt wird, sollte seine Arbeit noch in einer weiteren Hinsicht betrachtet werden, wofür man sich auf eine von Hammermayer vorgebrachte Charakterisierung des „deutschen Maurinismus“ berufen kann: Dieser besage „nicht mehr und nicht weniger [...], als daß das maurinische Vorbild für Genese, Methode und Thematik der Werke deutscher Benediktinerhistoriker entscheidend wurde ${ }^{\text {“143 }}$. Die Methode sei - für die Historia Frisingensis eingeräumt; hingegen bereiten „Genese“ und „Thematik“ erhebliche Schwierigkeiten. Die monographische Darstellung einer Bistumsgeschichte aus maurinischen Vorbildern herzuleiten, ist nicht ohne weiteres möglich. Wohl gab es entfernte Parallelen in der literarischen Produktion der Galli nostri, insbesondere die seit 1715 erscheinende Neubearbeitung der Gallia christi$a n a^{144}$, vielleicht auch noch Guy-Alexis Lobineaus Histoire de Bretagne ${ }^{145}$, die auch im Aufbau - ein Band Darstellung, ein Band Belegmaterial - der Historia Frisingensis nicht unähnlich ist, aber nichts weist darauf hin, dass Meichelbeck diese Werke kannte oder gar imitierte. Für eine nach Sedenzzeiten geordnete Geschichte eines Bistums gab es weit naheliegendere Vorbilder, zuletzt etwa, bereits mit intensivem Gebrauch von Urkunden, Nikolaus Schatens Annales Paderbornenses, die Meichelbeck nachweislich eingehend benutzt hat ${ }^{146}$. Auch das Jubiläum als Anlass der Geschichtsdarstellung war keine Idee, die aus Frankreich hätte importiert werden müssen ${ }^{147}$.

143 Hammermayer, Forschungszentren (wie Anm. 9) 165.

144 Denis de SAINTE-MARTHE et al., Gallia christiana in provincias ecclesiasticas distributa, 1 (Paris 1715).

145 Guy-Alexis Lobineau, Histoire de Bretagne, composée sur les titres et les auteurs originaux (2 Bde., Paris 1707); vgl. Maurice LeComte, Les Bénédictins et l'histoire des provinces aux $\mathrm{XVII}^{\mathrm{e}}$ et $\mathrm{XVIII}{ }^{\mathrm{e}}$ siècles. Revue Mabillon. Archives de la France monastique 17 (1927) 237-246; 18 (1928) 39-58, 110-133, 302-323, hier 18 39-43.

146 Nikolaus Schaten, Annalium Paderbornensium partes duae (2 Bde., Neuhaus 16931698). Ein Konvolut mit Notizen, das im Meichelbeck-Nachlass einem Konzept zum Chronicon Benedictoburanum beigebunden ist, tatsächlich aber überwiegend aus Vorarbeiten zur Historia Frisingensis besteht, enthält etliche Exzerpte aus Schaten: BStB München, Meichelbeckiana 8, 416v-417r, 422r-423v, 431r-v. Zum Material vgl. DACHS, Meichelbeckiana (wie Anm. 39) 194, mit irriger Zuweisung als ,Vorstudien“ zum Chronicon.

147 Ralph SchUller, Jubiläum, Fiktion oder zentenare Memoria? Zur retrospektiven Wahrnehmung der klösterlichen Jubiläumskultur, in: Das historische Jubiläum. Genese, Ordnungsleistung und Inszenierungsgeschichte eines institutionellen Mechanismus, hg. von Winfried MüLLER-Wolfgang FLÜGEL-Iris LOOSEN-Ulrich Rosseaux (Geschichte: Forschung und Wissenschaft 3, Münster 2004) 139-156; Franz QuARTHAL, Kloster Zwiefalten zwischen Dreißigjährigem Krieg und Säkularisation. Monastisches Leben und Selbstverständnis im 6. und 7. Saeculum der Abtei, 
Die genannten maurinischen Arbeiten waren im Übrigen die ersten Ausflüsse einer gerade erst einsetzenden Neuorientierung im Schaffen der Maurinergelehrten, die im weiteren Verlauf des Jahrhunderts zu erheblichen Aktivitäten in der Profan- und Territorialgeschichte führte ${ }^{148}$. Im zweiten und dritten Jahrzehnt desselben aber waren dies, gerade in der Wahrnehmung aus dem Ausland, noch Marginalien; die weithin bekannten und bewunderten Arbeiten der Mauriner waren neben den ordensgeschichtlichen Großwerken Mabillons, die mit seinem Tod ins Stocken gerieten, sowie seinem De re diplomatica vor allem - was in einer Mabillon-fixierten Historiographie der maurinischen Gelehrsamkeit zu wenig betont worden ist - die Kirchenväterausgaben $^{149}$. Die maurinische Produktion situierte sich damit im Zentrum einer gallikanischen kirchlichen Historiographie des späten 17. und beginnenden 18. Jahrhunderts, deren Fokus vor allem auf der älteren und ältesten Kirchengeschichte, auf der Geschichte des Mönchtums, der Liturgie, der kirchlichen Disziplin lag und die daher schwer war von theologischen und ekklesiologischen Implikationen ${ }^{150}$. Die Ergründung der Bräuche der frühen Kirche führte implizit oder auch offen zur Kritik an gegenwärtigen Zuständen und nicht zuletzt zum gallikanischen Widerstand gegen den Primat Roms.

Bei den am deutlichsten maurinisch beeinflussten benediktinischen „Forschungszentren" in Deutschland ist eine Anlehnung an die Werke der Mauriner in Thematik und Anlage klar zu erkennen. Bernhard Pez konzipierte

in: 900 Jahre Benediktinerabtei Zwiefalten, hg. von Hermann Josef PRETSCH (Ulm 1989) 401-430, hier 405-417, 423-428; SAWILLA, Antiquarianismus (wie Anm. 107) 225-227.

148 Gasnault, Mauristes (wie Anm. 15) 115-119; LAURAIN, Travaux d'érudition (wie Anm. 16) 237-250; LeCOMTE, Histoire des provinces (wie Anm. 145).

149 Daniel-Odon Hurel, Les mauristes, éditeurs des Pères de l'Église au XVII ${ }^{\mathrm{e}}$ siècle, in: Les Pères de l'Église au XVII ${ }^{\mathrm{e}}$ siècle. Actes du colloque de Lyon, 2-5 octobre 1991, hg. von Emmanuel BuRY-Bernard MEUNIER (Paris 1993) 117-134; Jean-Louis QuANTIN, Le catholicisme classique et les Pères de l'Église. Un retour aux sources (1669-1713) (Collection des Études Augustiniennes, Série Moyen-âge et Temps modernes 33, Paris 1999) 169-198.

150 Pierre ClAIR, Louis Thomassin (1619-1695). Étude bio-bibliographique avec vingt lettres et deux textes inédits (Le mouvement des idées au XVII ${ }^{\mathrm{e}}$ siècle 1, Paris 1964); Bruno Neveu, Un historien à l'école de Port-Royal. Sébastien Le Nain de Tillemont 1637-1698 (Archives internationales d'histoire des idées 15, La Haye 1966); Bruno NEVEU, Mabillon et l'historiographie gallicane vers 1700. Érudition ecclésiastique et recherche historique au XVII ${ }^{\mathrm{e}}$ siècle, in: Historische Forschung im 18. Jahrhundert (wie Anm. 9) 27-81; Jean-Louis QUANTIN, Document, histoire, critique dans l'érudition ecclésiastique des temps modernes. Recherches de science religieuse 92 (2004) 597-635. Zur Verbindung zwischen historischer Gelehrsamkeit, positiver Theologie und Ekklesiologie vgl. auch die Beiträge von Jean-Louis Quantin und Bernward Schmidt in diesem Band. 
seine unfertig gebliebene Bibliotheca Benedictina generalis ausdrücklich als Ergänzung zu Mabillons Annales und Acta, seine Quellenedition Thesaurus anecdotorum novissimus, in welcher mittelalterliche monastische Theologie und Lebensweise als Vorbild zur Erneuerung in der Gegenwart präsentiert wurden, orientierte sich an Edmond Martènes fast gleichnamiger Publika$\operatorname{tion}^{151}$. Marquard Herrgott veröffentlichte, bevor er sich der Genealogie der Habsburger zuwandte, eine Quellenpublikation zur Vetus disciplina monas$t_{i c a}{ }^{152}$. Auch spätere Projekte in diesen Klöstern, etwa Frobenius Forsters Alkuin-Ausgabe oder Martin Gerberts Geschichte der Kirchenmusik ${ }^{153}$, passen in dieses Schema stark theologisch orientierter universeller Ordensund Kirchengeschichte besser als die Historia Frisingensis oder auch Meichelbecks letztes großes Werk, das Chronicon Benedictoburanum, auf das hier nicht näher eingegangen werden kann.

Letztlich wird man die Motivationslage von Meichelbecks historiographischer Tätigkeit nicht als besonders „maurinisch“ bezeichnen können. Die partikulare Ehre der Institutionen, für die und über die er schrieb, stand bei ihm wohl stets im Zentrum und vor der Perspektive auf die Kirche als Ganzes oder auch auf das Reich oder das Land. Rechtstitel und ihre Verteidigung spielten für ihn, dessen Werdegang immer wieder von Rechtsstreitigkeiten und der Abfassung von Deduktionen geprägt war, eine zentrale Rolle; seine sich verfestigende Überzeugung von der erstrangigen Beweiskraft urkundlicher Belege hatte ihren Hintergrund wohl nicht zuletzt darin, dass er sie oft tatsächlich - und meist mit Erfolg - vor Gericht vorgebracht hatte ${ }^{154}$. Seine Liebe zum Benediktinertum äußerte sich nicht vorrangig im Bestreben, das Mönchtum aus der Vergangenheit heraus zu reformieren, sondern vor allem in der Verteidigung seines Ordens gegen andere, seien es die Augustiner-Chorherren ${ }^{155}$ oder die Jesuiten ${ }^{156}$. Theologisch hielt sich der

151 Edmond Martène-Ursin Durand, Thesaurus novus anecdotorum (5 Bde., Paris 1717); Pez, Thesaurus (wie Anm. 134); vgl. Glassner, Thesaurus (wie Anm. 134) 343f., 348f.; Wallnig, Pez und Mauriner (wie Anm. 19) 158f., 162-164.

152 Marquard HerrgotT, Vetus disciplina monastica seu Collectio auctorum ordinis sancti Benedicti maximam partem ineditorum, qui ante sexcentos fere annos per Italiam, Galliam atque Germaniam de monastica disciplina tractarunt (Paris 1726); vgl. ORTnER, Herrgott (wie Anm. 46) 20-24.

153 Beati Flacci Albini seu Alcuini abbatis, Caroli Magni regis ac imperatoris magistri, Opera, hg. von Frobenius Forster (4 Bde., Regensburg 1777); Martin GERBERT, De cantu et musica sacra a prima ecclesiae aetate usque ad praesens tempus (2 Bde., St. Blasien 1774).

154 WÜHR, Bedeutung (wie Anm. 27) 230, spricht nicht zu Unrecht davon, Meichelbeck sei ,unversehens [...] vom Juristen zum Historiker geworden“. Nicht gefolgt werden kann ihm jedoch, wenn er hierin eine direkte Parallele zu Mabillon sehen will.

155 Vgl. Anm. 77, 80, 92. Noch 1728 gefiel sich Meichelbeck darin, Amort die Erhebung 
Neuerungswille Meichelbecks in Grenzen: Sein resoluter Antischolastizismus und sein Interesse an historischen Studien führten ihn nicht zu Zweifeln an der päpstlichen Autorität - wie er selbst so treffend sagte, hielt er es im Gegensatz zu den Maurinern im Zweifel mit dem Papst gegen die alten Handschriften. Auch an der Barockfrömmigkeit ließ sich der lebenslange glühende Verehrer der Hl. Anastasia nicht irre machen ${ }^{157}$.

\section{Schlussbemerkungen}

Letzten Endes kann die Frage, ob Meichelbeck ein „deutscher Mauriner“ gewesen sei, wohl nicht absolute beantwortet werden, sondern nur secundum quid. Er war einer in der engsten Interpretation des Hammermayer'schen Konzeptes: ein deutscher Benediktiner, der sich die Grundsätze kritischer Geschichtsforschung zu eigen machte. Mit jeder Erweiterung der Definition wird sie aber schwieriger auf ihn anzuwenden. Was bleibt von dem „führenden Repräsentanten“158 des „deutschen Maurinismus“? Ein Mann, dessen direkte Kontakte mit der Maurinerkongregation bestenfalls punktuell, vielleicht aber auch inexistent waren; ein Mönch, dessen gelegentlichen maurinerfreundlichen Äußerungen ${ }^{159}$ ein Handeln nach deren Vorbild nicht in ähnlichem Maße entsprach wie bei seinen Ordensbrüdern in St. Emmeram, St. Blasien oder Melk; ein Gelehrter, für den Historiographie wohl weniger als für Bernhard Pez „als Geschichte betriebene Theologie ${ }^{\text {“160 }}$ war, dafür

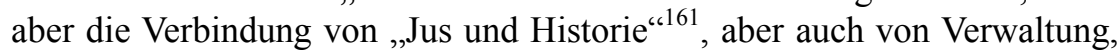
Archivpraxis und Geschichte, ein umso größeres Gewicht hatte.

eines Benediktiners zum Kardinal vorzuhalten, als jener Benediktbeuern besuchte: BStB München, Meichelbeckiana 18c, 225v (18. Mai 1728).

156 Vgl. Siegmund, Meichelbecks Briefe (wie Anm. 43) 80/1-2 150-152 Nr. 19, $154 f$. Nr. 21, 157-160 Nr. 23; WALlNiG-StOCKINGER, Korrespondenz 1 (wie Anm. 43) 755-761 Nr. 464, 775-780 Nr. 474; WeBER, Meichelbeck (wie Anm. 20) 134f.

157 Vgl. WeBER, Meichelbeck (wie Anm. 20) $137 \mathrm{f}$.

158 Vgl. Anm. 34.

159 Vgl. Anm. 75; sowie den Brief an Bernhard Pez vom 4. Oktober 1716: SiEGMUND, Meichelbecks Briefe (wie Anm. 43) 80/1-2 162-164 Nr. 25; WeBER, Meichelbeck (wie Anm. 20) 132-134. Daraus herleiten zu wollen, bei Meichelbeck könne man „von einer Franco-Bavarico-Austriaco-Allianz sprechen“ (ebd. 133), ist jedoch auch abgesehen vom Stilistischen - reichlich fragwürdig.

160 Thomas Wallnig, Gasthaus und Gelehrsamkeit. Studien zu Herkunft und Bildungsweg von Bernhard Pez OSB vor 1709 (Veröffentlichungen des Instituts für Österreichische Geschichtsforschung 48, Wien-München 2007) 172.

161 Vgl. Notker Hammerstein, Jus und Historie. Ein Beitrag zur Geschichte des historischen Denkens an deutschen Universitäten im späten 17. und im 18. Jahrhundert (Göttingen 1972). 
Dies lädt dazu ein, abschließend zu fragen, wie weit das Konzept eines „deutschen Maurinismus“ trägt und hilfreich ist. Angesichts dessen, in wie vielen Hinsichten sich deutsche Benediktiner an den Maurinern orientieren konnten, aber auch des Umstands, dass diese einzelnen Formen und Aspekte der Auseinandersetzung mit ihnen keineswegs notwendig alle gebündelt auftraten, wäre es vielleicht sinnvoller, von verschiedenen Spielarten der Maurinerrezeption zu sprechen, als einen einheitlichen „Maurinismus“ $\mathrm{zu}$ postulieren. Diese Rezeptionsprozesse in ihrer ganzen Variationsbreite, ihren zeitlichen Veränderungen und natürlich auch in ihren Ausprägungen außerhalb des Benediktinerordens, wovon das meiste in diesen Ausführungen nicht einmal berührt werden konnte, sind weiterhin ein lohnendes Forschungsgebiet. Skepsis scheint allerdings angebracht gegenüber einer Definition von „Maurinismus“, die ihn mit der Verbreitung der historisch-kritischen Methode im benediktinischen Milieu gleichsetzt. Sie ist in doppelter Weise reduktionistisch: Einerseits reduziert sie die Maurinerkongregation auf Protagonisten einer geschichtswissenschaftlichen Innovation, was deren Selbstverständnis ebenso wenig gerecht wird wie der gesamten Bandbreite ihrer Außenwirkung. Andererseits impliziert sie, zumindest für die Benediktiner, den alleinigen Ursprung dieser Methode bei den Maurinern. Das Beispiel Meichelbecks, das vor Augen führt, wie wenig „maurinisch“ ein Spitzenvertreter dieser Methode in anderen Hinsichten war, sollte ausreichen, um dies in Frage zu stellen und zur Suche nach weiteren Traditionssträngen anzuregen, aus denen sie sich konstituierte.

\section{Abstract}

First coined by Paul Muschard, the term "German Maurism" was not raised to the status of a paradigm for the understanding of monastic and especially Benedictine historical scholarship in Catholic southern Germany until fifty years later by Ludwig Hammermayer. He defined it as the abandonment of Baroque historiography, which he associated with „,compilation, apology, and triumphalism", in favour of the systematic collection of sources and their use in analytic reconstruction of the past. This narrow definition of „Maurism“, which essentially equated it with the spread of the historical-critical method within the monastic milieu, was expanded by later researchers to include other aspects of Benedictine life, leading to the reconstruction of a „Maurist reform programme" in which scholarly activity appears as a component of an encompassing renewal of monasticism, pursued in German Benedictine abbeys following the example of the French congregation of Saint-Maur.

Karl Meichelbeck (1669-1734) of the abbey of Benediktbeuern, archivist and historian, was the author of the Historia Frisingensis, a history of the 
bishopric of Freising published in 1724-1729. The work has often been classified in the history of historiography as a milestone in the dissemination of the „Maurist method“, and Meichelbeck himself is among the handful of scholars who have received the sobriquet ,the German Mabillon“": a superlative, if one will, of "German Maurist"?

The present article re-examines this equation in several ways. On the one hand, it attempts to trace Meichelbeck's actual connections to the Maurists: his use of their works, contacts by way of correspondence, references to them in his writings. These prove to be quite sparse in comparison with other Benedictine "centres of research" such as Melk or St. Emmeram's in Ratisbon. On the other hand, the paper sketches the development of Meichelbeck's scholarly technique on the basis of selected passages from his writings. The Historia Frisingensis and his other main work, Chronicon Benedictoburanum, appear not at the beginning but at the end, as the achievements of his old age. How Meichelbeck became a critical historian - a question which has already frustrated previous biographers - cannot be answered definitively, but some clues are presented. That Meichelbeck the critical historian was not necessarily also Meichelbeck the „German Maurist“" leads to concluding reflections on the value and weaknesses of the latter term as a paradigm for further research. 